\title{
A Survey of Geometric Analysis in Cultural Heritage
}

\author{
Ruggero Pintus ${ }^{1} \quad$ Kazim Pal $^{3} \quad$ Ying Yang $^{2} \quad$ Tim Weyrich $^{3} \quad$ Enrico Gobbetti $^{1} \quad$ Holly Rushmeier $^{2}$ \\ ${ }^{1}$ CRS4 $\quad{ }^{2}$ Yale University $\quad{ }^{3}$ University College London
}

\begin{abstract}
We present a review of recent techniques for performing geometric analysis in cultural heritage applications. The survey is aimed at researchers in the areas of computer graphics, computer vision, and cultural heritage computing, as well as to scholars and practitioners in the cultural heritage field. The problems considered include shape perception enhancement, restoration and preservation support, monitoring over time, object interpretation, and collection analysis. All of these problems typically rely on an understanding of the structure of the shapes in question at both a local and global level. In this survey, we discuss the different problem forms and review the main solution methods, aided by classification criteria based on the geometric scale at which the analysis is performed and the cardinality of the relationships among object parts exploited during the analysis. We finalize the report by discussing open problems and future perspectives.
\end{abstract}

\section{Introduction}

Computing in Cultural Heritage $(\mathrm{CH})$ has become a wellknown and broad research field. Databases and text data mining for heritage applications have been lively and productive areas of computing. Among those topics, the use of computer graphics techniques in cultural heritage has been an active area of research for over the past 20 years. Advances have been made from several points of view, so that the use of graphics in cultural heritage has now grown too large to survey as a whole. While there are many ways its contributions can be categorized, one broad scheme is to consider three classes - acquisition, communication and analysis.

Many research projects have focused on the acquisition of 3D models with cultural heritage as a motivating application, going back to the 1980's [BRTL88]. Numerous surveys have been produced for the acquisition [REH06]. Cultural heritage has some special requirements, such as limitations on touching or temporarily modifying the surface (such as by spraying with white powder), in addition to frequent financial constraints. Novel acquisition methods for cultural heritage have progressed substantially, and have proved useful for acquisition for other purposes.

Many projects have also focused on the use of graphics for illustration and public communication in cultural heritage, with surveys of these efforts already appearing more than 10 years ago [Add00]. These projects draw on and contribute to techniques in multimedia, human interaction and web technology. Topics such as augmented reality, mobile and gaming technologies continue being explored for presentation and public engagement.

In this review, rather than considering acquisition and communication, we survey the analytical techniques that are facilitated by computer graphics after the relevant models have been acquired. In particular, we focus our attention on the vast field of analytical techniques applied to geometry that have been developed for heritage applications. These techniques are directed at experts in archaeology, history and/or conservation, rather than for communication with or engagement of the general public. Analysis is performed to answer typical questions in $\mathrm{CH}$, such as "how was this made?", "why was this object made this way?", "who made this?", "how has this changed through time?", and "how can this object best be protected from damage?".

We hope that this comparative survey will help readers navigate through the constantly expanding literature on geometric analysis in cultural heritage. The target audience of our report includes computer graphics, computer vision, and cultural heritage computing researchers, as well as scholars and practitioners in the cultural heritage field. Researchers will find a structured overview of the field that organizes the various methods and their applications to $\mathrm{CH}$ in a taxonomy 
and indicates challenging open problems. Domain experts will, in turn, find a presentation of the areas where geometric analysis is helping, or has the potential to improve, scholarly research in cultural heritage.

This work is an invited extended version of our Graphics and Cultural Heritage 2014 contribution [PPY*14], that presented a survey of methods by considering a classification with respect to the geometric scale at which the analysis is performed, the number of objects involved and the cardinality of the relationship between them, and the cultural heritage application to which the analysis is applied. In addition to supplying a more thorough exposition, in this work we also provide significant new material, including additional references and examples, a stronger and sharper definition of our classification, especially with respect to geometric scale, a review of the multi-scale works that use a weighted combination of two or more levels of analysis (Sec. 7), and an improved discussion on the distribution and clustering of papers in the literature in order to better highlight possible directions for future works.

The rest of this survey is organized as follows. In Sec. 2, we provide pointers to other closely related surveys on computer-based techniques applied to cultural heritage. Sec. 3 introduces our taxonomy of geometric analysis in cultural heritage and provides a global overview of the literature. The main part of the survey then examines the various approaches that work predominately at a micro- (Sec. 4), meso- (Sec. 5), and macro- (Sec. 6) geometric scale. We then discuss hybrid methods (Sec. 7) that use multiple geometric scales together for their analysis. We conclude the survey with an analysis of the distribution of the various approaches depending on the cultural heritage applications and with thoughts on future challenges in the field (Sec. 8).

\section{Related surveys}

The area of geometry analysis is vast and has many applications. We focus specifically on the field of cultural heritage, discussing the techniques that have found practical applications in this field. We refer the reader to more general works (e.g. [SS01, MPWC13, MWZ*14]) for domain-independent surveys of the subject.

A number of surveys have been presented that relate to the general subject of computer-based techniques applied to Cultural Heritage. We draw on these surveys which in some cases treat subsets of what can be considered as analysis, and in some cases treat supersets of issues that include analysis.

Owen et al. [OBP04] present a systematic study to identify the technologies used in the cultural heritage field from papers presented at VAST 2003, adopting an information systems theory approach that classifies procedures into three activities: input, process or output. They also outline the trends in technologies used within cultural heritage.
Happa et al. [HMD*10] review the current state of illuminating cultural heritage sites and objects using computer graphics. While the book edited by Stanco et al. [SBG11] covers digital imaging-driven cultural heritage preservation, Ask [Ask12] investigates and discusses possible methods of 3D documentation methodology and interpreting $\mathrm{CH}$ data through computer vision techniques. More broadly, Stork [Sto09] briefly summarizes how the computer vision, image analysis and computer graphics algorithms have been used to process and understand digital images of scanned art, particularly paintings and drawings.

Rather than giving a comprehensive survey of all recent computer graphics techniques used for Cultural Heritage, Scopigno et al. [SCC ${ }^{*} 11$ ] present some examples to show the significant impact of digital 3D models on the daily work of $\mathrm{CH}$ scholars, curators, and restorers, and highlight some important applications of analysis in cultural heritage, including reassembly, attribution and monitoring.

\section{Overview}

The field of geometric analysis in cultural heritage is very large, and covers all applications in which the shape of cultural objects, parts of them, or collections of them, carries valuable qualitative and quantitative information that is used as a basis for analysis tasks. We provide a structured view of this large field by introducing a taxonomy of methods by considering three axes of classification, i.e., Geometric Scale at which the analysis is performed, number of objects (\# Objects) involved in the analysis and the relationship between them, and the $\mathbf{C H}$ application that employs the analysis to answer specific questions (Fig. 1).

In order to classify these approaches according to the proposed taxonomy, we consider their predominant scale of analysis, before reviewing in a dedicated section (Sec. 7) the most relevant multi-scale algorithms, linking them to the other geometric scale classes.

For each class we explain the nature of the 3D data used, and we classify the methods according to the number of objects (\# Objects) that participate to the analysis. In particular, we group methods in three classes: methods that study a single object, methods that compare one object in relation with a set of similar samples, and methods that perform a many-to-many comparison.

Finally, we classify methods according to the $\mathbf{C H}$ application in which the analysis is typically performed. We have identified the following classes: perception enhancement, restoration and preservation, monitoring, interpretation and collection analysis. In Table 1 we summarize the distribution of the works in the literature related to our proposed taxonomy. 


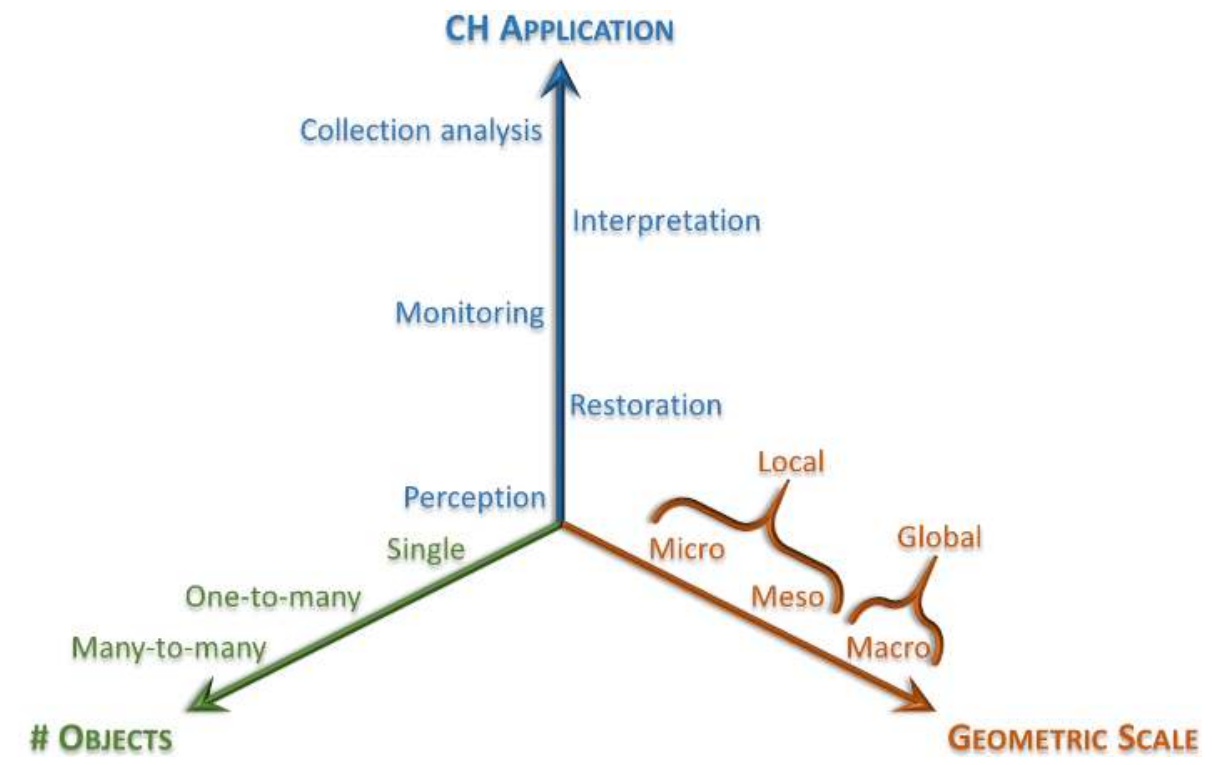

Figure 1: Taxonomy. We define our taxonomy based on three classification axes: Geometric Scale at which the analysis is performed, \# Objects involved, and $\mathbf{C H}$ application.

\section{Micro geometry}

In this section we organize the literature related to the techniques that exploit small geometric features to perform analysis in the $\mathrm{CH}$ field.

Finally, these innovative methodologies have been designed, implemented and assessed to follow the specific needs of real $\mathrm{CH}$ problems, and they are possible due to the accuracy of the 3D scanned models acquired nowadays. In the last decade, close-range 3D scanners based on laser triangulation or structured light have been developed through intensive research, both academic and industrial, and they are able to produce very dense models with up to a submillimeter resolution.

\subsection{Single object analysis}

Perception enhancement. Geometric operators at fine scale are typically applied to high-resolution 3D models, to analyze and extract small structures, and enhance the details, such as impressed characters or other high-frequency signals that are difficult to perceive. These approaches typically use differential geometry coupled with multiscale frameworks. Mara et al. [MKJB10, MK13] propose a transformation invariant feature vector, that is obtained by calculating the volumes of multiple concentric spheres intersecting the volume below the 3D model's surface at each point. They increase the readability of 3D scanned cuneiform tablets (Fig. 2), and use this signal to produce artificial illustrations of the writing (Fig. 3). Kolomenkin et al. [KST11] define a new direction field on surfaces (i.e., prominent field) that is ori-

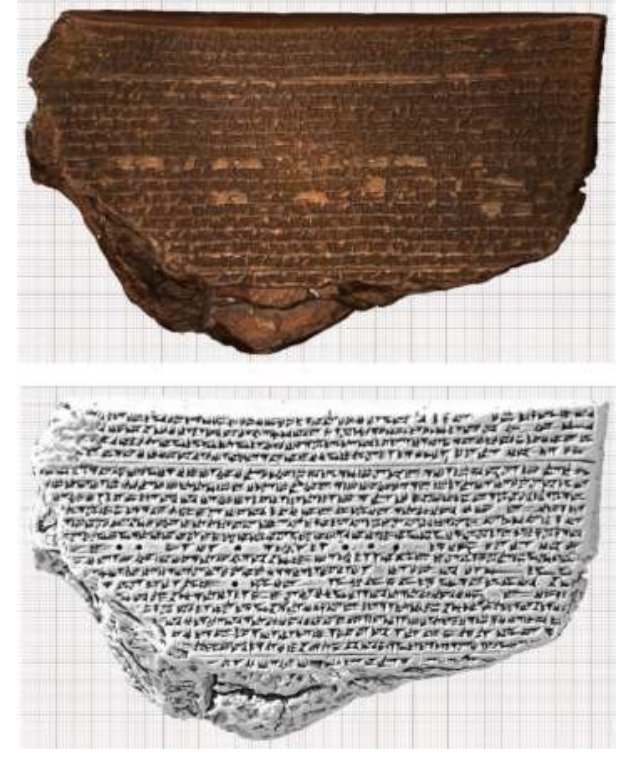

Figure 2: Increasing the readability of $3 D$ scanned cuneiform tablets. 3D model of Inv. No. W51617 of the Assyriologie Heidelberg collection. Top: The acquired texture mapped sample. Bottom: the gray-scale colored model after applying the perception enhancement technique. Courtesy of Mara et al. [MKJB10].

ented with respect to the prominent feature curves of the surface; they use this operator both for surface enhance- 
Table 1: Geometric Analysis Taxonomy.

\begin{tabular}{|c|c|c|c|c|}
\hline Scales \Objects & Single Object & One-to-many & Many-to-many & $\begin{array}{l}\mathrm{CH} \text { Applica- } \\
\text { tion }\end{array}$ \\
\hline \multirow[t]{5}{*}{ Micro-scale } & $\begin{array}{l}\text { [CGPS08, GTSK13, GWS*09, } \\
\text { HW11, KST08, KST11, } \\
\text { LPC }^{*} 00, \text { MGW01, MKJB10, } \\
\text { MK13, MVSL05, MMSL06, } \\
\text { PGCS10] }\end{array}$ & & & Perception \\
\hline & & & 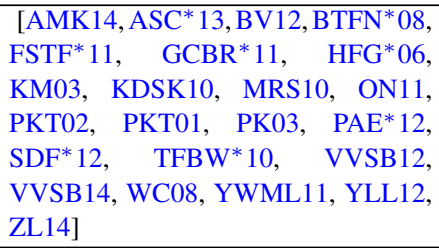 & Restoration \\
\hline & {$\left[\mathrm{BCP}^{*} 07, \mathrm{MBW}^{*} 14\right]$} & & & Monitoring \\
\hline & & & & Interpretation \\
\hline & & & & $\begin{array}{l}\text { Collection } \\
\text { Analysis }\end{array}$ \\
\hline \multirow[t]{5}{*}{ Meso-scale } & $\begin{array}{l}\text { [PTW13a, PTW13b, PSP*14, } \\
\text { PMCS11, SSB11] }\end{array}$ & & & Perception \\
\hline & [PMW*09] & & $\begin{array}{l}\text { [CFB }{ }^{*} 13, \text { CLE12, KS04, KO13, } \\
\text { LW04a, LYW }^{*} 11, \text { TFK }^{*} 09, \text { WYLL11, } \\
\text { WC04a, WC04b, WC08] }\end{array}$ & Restoration \\
\hline & & & & Monitoring \\
\hline & [AIK13] & & & Interpretation \\
\hline & & $\begin{array}{lr}\text { [IT11, KS08, KS11, } \\
\text { KPC10, } \\
\text { SPK }{ }^{*} 13, & \text { SPK }^{*} 14, \\
\text { SKN*12] } & \\
\end{array}$ & & $\begin{array}{l}\text { Collection } \\
\text { Analysis }\end{array}$ \\
\hline \multirow[t]{5}{*}{ Macro-scale } & $\begin{array}{c}\text { [BKMK10, } \\
\text { PBM*13, } \\
\text { PCMA08, RMK13] }\end{array}$ & & & Perception \\
\hline & $\begin{array}{l}\quad\left[\mathrm{BS} 04, \mathrm{BSY}^{*} 07, \mathrm{CCG}^{*} 04,\right. \\
\text { FdCP* }^{*} \text { 08, FMOS11, HRM*09, } \\
\text { LSC08, Lan13, LD06, LLS06, } \\
\text { PSP*14, SEG*12, SLMR14, } \\
\text { SL04, SYY*05, WCV*03] }\end{array}$ & [LS09] & {$\left[\right.$ LZT $\left.^{*} 11\right]$} & Restoration \\
\hline & 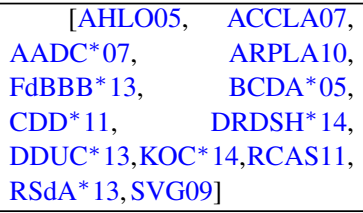 & {$\left[\mathrm{FRB}^{*} 13\right]$} & & Monitoring \\
\hline & $\begin{array}{l}\quad\left[\mathrm{DCF}^{*} 07, \text { DST05, DB06, }\right. \\
\text { LCM11, MGM*12, NFRM15, } \\
\text { DACC05] }\end{array}$ & & & Interpretation \\
\hline & & {$\left[\mathrm{BP} 10, \mathrm{GCJ}^{*} 07\right]$} & [BLMT*14, LZZ*13,ZLZ*13] $^{*}$ & $\begin{array}{l}\text { Collection } \\
\text { Analysis }\end{array}$ \\
\hline
\end{tabular}

ment of archaeological artifacts, and for artificial coloring that can replace manual artifact illustration in archaeological reports. In another paper, Kolomenkin et al. [KST08] propose a new class of view-independent curves (demarcating curves) to increase readability of shapes and details in the visualization and rendering of $3 \mathrm{D}$ digital artefacts. These curves represent the regions of strongest inflection of the surface geometry, and are used to produce artificial illus- tration and increased quality, meaningful illumination, by coupling them with mean-curvature or exaggerated shading. Similarly, Gilboa et al. [GTSK13] use micro-scale feature computation to translate 3D scanned models of artifacts into different kind of representations, such as 3D or 2D line drawings, and colored images that highlight primitive saliency and enhance detail perception. Their aim is to use new algorithms specifically designed to replace traditional, manual il- 
R. Pintus, K. Pal, Y. Yang, T. Weyrich, E. Gobbetti, H. Rushmeier / Geometric Analysis in Cultural Heritage
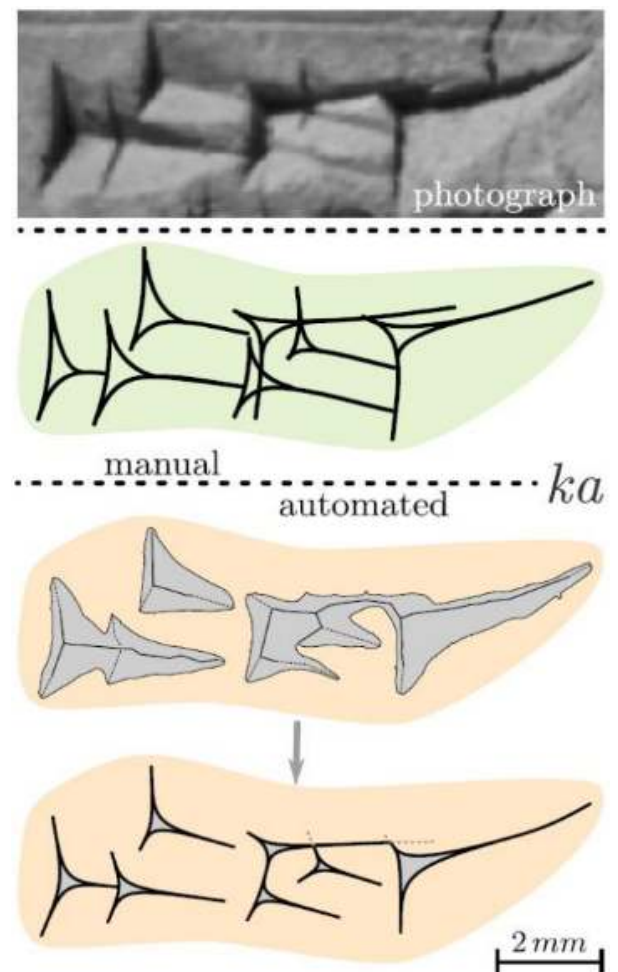

Figure 3: Artificial illustration. The comparison between manual drawing of the character ka and the vector artificial illustration computed from the 3D-model. Courtesy of Mara et al. [MK13].
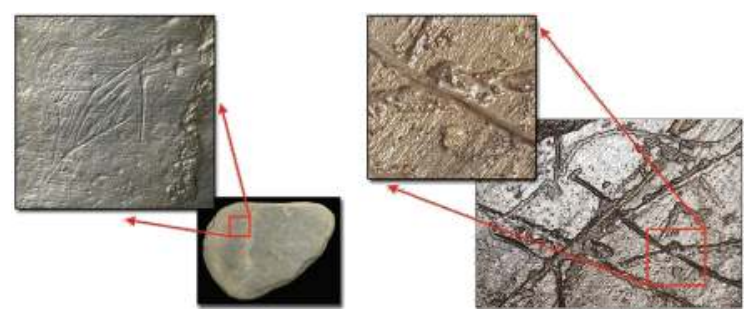

Figure 4: PTM-based perception enhancement: rock art. Improvement of the readability of petroglyphs by applying specular enhancement to the normal field computed from RTI data. Courtesy of Mudge et al. [MMSL06].

lustrations, which are very expensive and prone to human errors. This work tries to address issues and improve methodologies in the ever increasing complexity and innovation of modern research in archaeology. Levoy et al. [ $\left.\mathrm{LPC}^{*} 00\right]$ use accessibility shading [Mi194] to visually enhance the appearance of chisel marks in 3D reconstructions of sculptures by Michelangelo. Polynomial Texture Map (PTM) [MGW01] and Photometric Stereo (PS) [Woo79] are other techniques that use the per-vertex computation of surface normals to

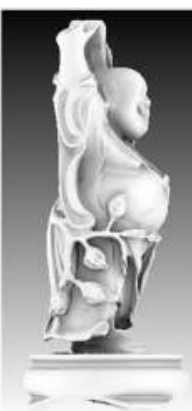

(a)

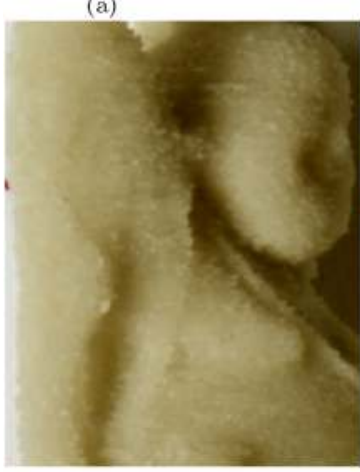

(c)

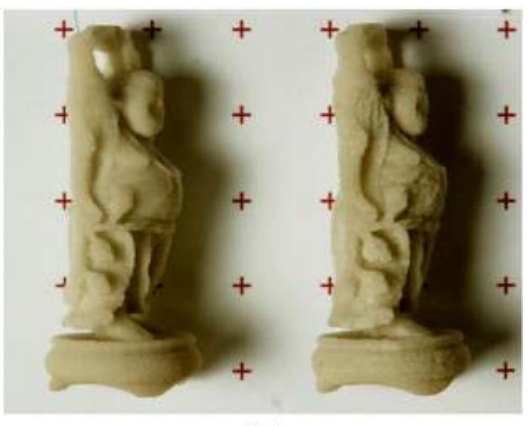

(b)

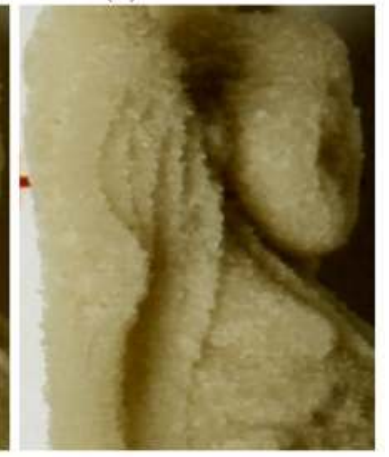

Figure 5: Shape enhancement. A $3 D$ model of Buddha statuette [Aim03]: (a) the original model lit with ambient occlusion; (b) the printed replicas of the original and geometrically enhanced models; (c) is the comparison between a small part of the original and enhanced model. In the latter, more details are better perceivable and preserved [PGCS10].
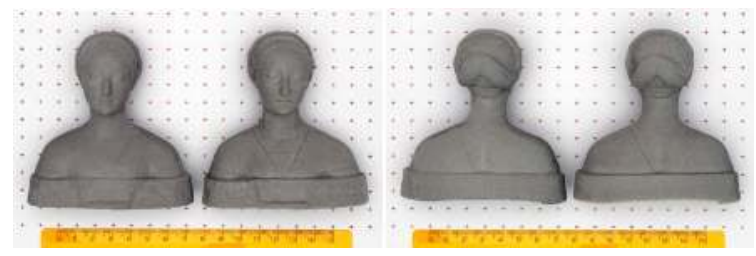

Figure 6: Shape perception enhancement through color modification. The color-enhancing technique in action on the Laurana model [Aim03]. Represented objects are less than $10 \mathrm{~cm}$ tall (crosshairs are $1 \mathrm{~cm}$ spaced). In each pair of images, the left model is a standard uniform color reproduction, while on the right the same 3D model has been printed with enhanced color: many small geometric details whose shading is canceled by the sub-surface scattering effect are again visible in the model on the right [CGPSO8].

increase the perception of fine geometric details in $\mathrm{CH}$ visualization and analysis. Hameeuw and Willems [HW11] apply PTM to increase visualization of cuneiform tablets and 
sealings. For instance, the acquisition and characterization of coin surfaces [GWS*09, MVSL05] overcome visual limitations of traditional documentary techniques and provides an improved tool for numismatic understanding and coin morphology analysis. The incomplete nature of rock art drawing and photography data makes them difficult to read. Instead, PTM proved to be an efficient way to enhance the perception of petroglyphs. Mudge et al. [MMSL06] show how the use of PTM on Paleolithic rock art allows scholars to better discern the most difficult to see surface attributes and fine details.

The enhancement of small detail perception is also very important in rapid prototyping. Pintus et al. [PGCS10] developed a geometry enhancement operator that counterbalances the effects due to the non-ideal behavior of the materials used in the printing process (Fig. 5). They represent the geometry as a volume, and they simulate on this volume the physical behavior of the printer. Then they compare the result to the original geometry, and modify the input data in order to reduce the difference between the original model and the printed one. Their function aims at locally increasing the contribution of geometrical high frequencies. A similar approach was presented by Cignoni et al. [CGPS08]. Instead of modifying the geometry, they exploit the color painting capabilities of recent 3D printers, and propose a technique to color the surface of an object in order to enhance the perception of its geometric details (Fig. 6); they analyze the fine scale geometry to compute a counter shading signal, which is "baked" in the color attribute of each vertex before the 3D print. This approach overcomes the drawbacks of subsurface scattering effects of the printing material, which usually blurs out the perception of the shades of the smallest details. 3D printing technology can also be used in a straightforward manner to produce replica at higher scales, or in preservation applications, such as transporting or storing $\mathrm{CH}$ artifacts (see section 6.1).

Monitoring. A general problem in $\mathrm{CH}$ is the measurement of preservation strategies by monitoring the micro-geometric changes over time. Performing repeated 3D acquisitions on a time schedule is useful for tracking fine deformations and degradation of artwork. Brunetti et al. [BCP* 07] performed a periodic 3D scanning campaign, and showed how to measure, compare and monitor wood deformations caused by weather changes in the trees of the fossilized Dunarobba forest. They study different conservation methodologies, such as covering individual trunks with artificial roofing, maintenance of some trunks inside a climatic chamber, or surface coating of trunks by an impregnating resin product. For each area of interest they performed four 3D acquisitions with an interval of three months. They measure the modification of the area in terms of Hausdorff distance between each pair of scans, and propose the choice of the best conservation methodology with a measurable evidence. The computation of normals by the PTM technique and the RTI acquisition setup has been used to detect morphological changes over time in the surface of paintings [MBW*14]. This non-invasive, high-resolution and powerful method is able to spot both geometrical changes as holes, bends, and cracks, and color modifications like abrasion of the original canvas. The authors demonstrated that they can even extract changes smaller than one half millimeter.

Interpretation. The relief and contour lines on a group of ancient Greek red-figure vases and vase fragments are examined with the Polynomial Texture Map (PTM) [MGW01], Photometric Stereo (PS) [Woo79] and 3D scanning for interpretation purposes [AIK13]. They characterize the shape of lines in artworks and answer questions regarding tools, techniques, and production sequences used by Greek vase painters. They find two distinct types of relief lines, and give a better understanding of the ancient technology. Further, the work confirms that the relief lines were not produced by an extruded method, but with a brush made with only a few hairs.
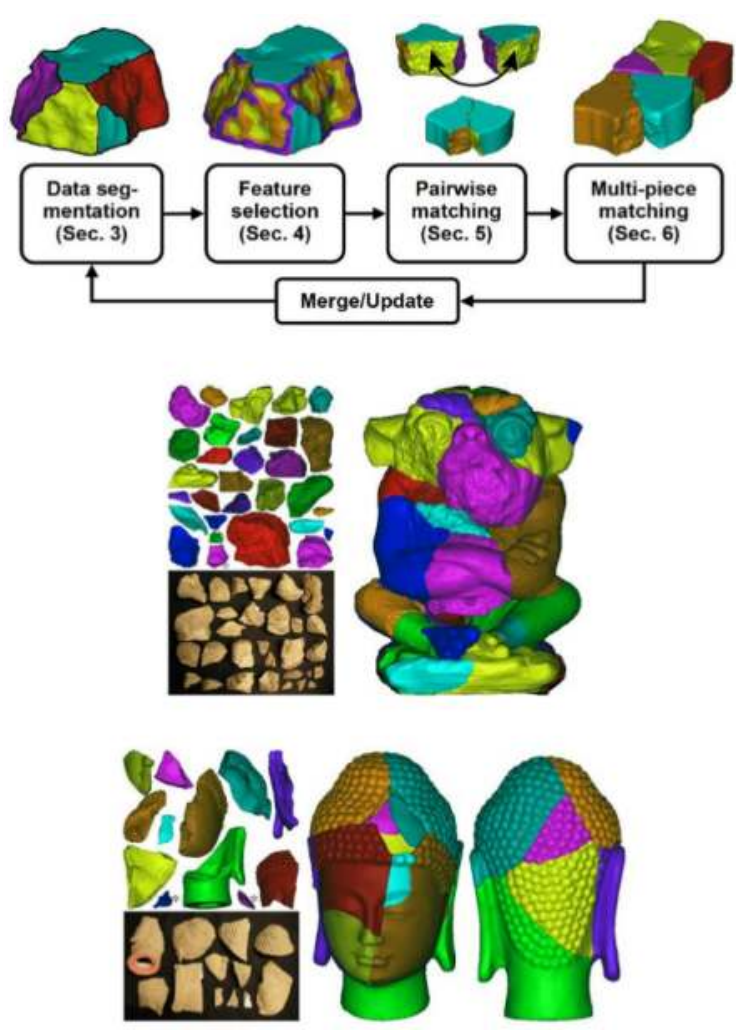

Figure 7: Reassembling fractured objects. Top row: high level overview of the reassembly pipeline. Middle and bottom row: two examples of reassembled objects. For each object the image shows the photo (bottom left) and the $3 D$ models (top left) of the fragments, and the final assembly (right). Courtesy of Pottmann et al. [HFG* 06]. 


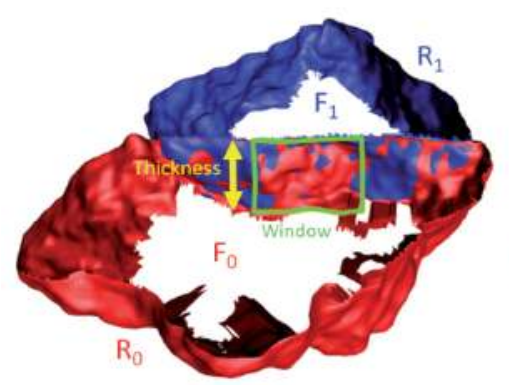

(a) aligned ribbons

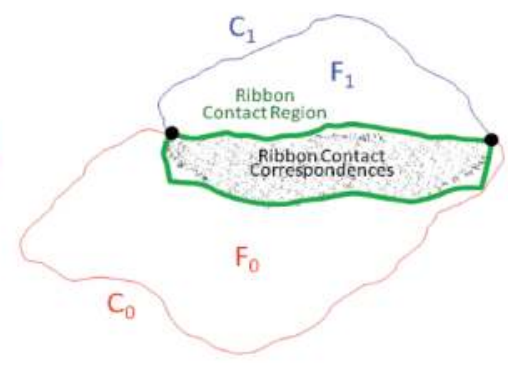

(b) ribbon contacts

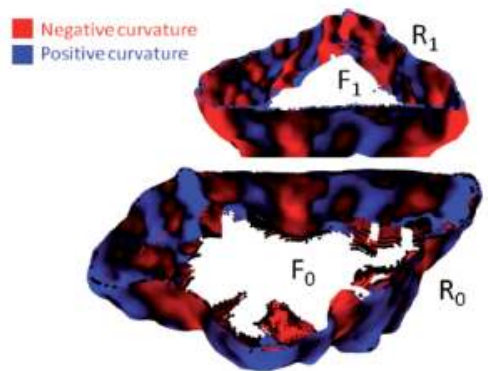

(c) ribbon curvatures

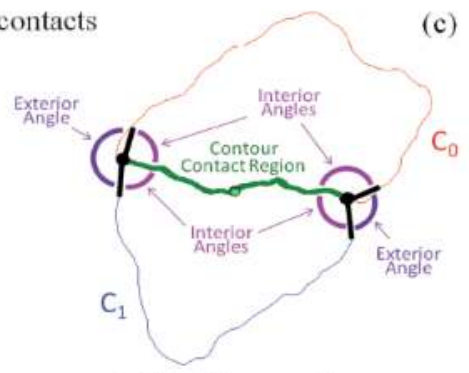

(d) contour overlaps

(e) junction angles

Figure 9: Geometric features in fragment re-assembly. Example of micro-scale measurements utilized by match properties in fragment re-assembly pipelines [FSTF* $\left.{ }^{*} 11\right]$.

\subsection{Many-to-many analysis}

Restoration and preservation. Micro-scale analysis and feature extraction have proven to be a central part of reassembly applications, which address the reconstruction of fractured physical artifacts from their pieces. The pieces have generally been scattered over time, so that no a priori information is available about the whole, final, reconstructed geometry. These applications make use of various types of data - primarily geometric data, but texture and surface properties are also used. Many methods aim to be fully automatic, while others make use of user input.

While also related to computational geometry, the reassembly of fractured objects is mostly treated as a patternmatching problem, and respective computer vision and graphics technologies have successfully been applied for reconstructing artifacts from fragments. The main idea is to find corresponding parts, to match and align them, and to finally reassemble all matching parts. Most of the existing approaches have focused on matching and analysis of the break boundary curves of fragments as they are visually important clues for reconstruction [PKT02, $\mathrm{HFG}^{*} 06, \mathrm{BTFN}^{*} 08$, YLL12]. By indexing features of the geometric information contained in the fracture surfaces of the fragments, Huang et al. $\left[\mathrm{HFG}^{*} 06\right]$ present a method for automatic reassembly of broken 3D solids Fig. 7. Yu et al. [YLL12] employ a multi-scale descriptor and conduct heat kernel-based partial matching between break boundaries, while Vendrell et al. [VVSB12] use hierarchical depth maps to ensure global correction of the reconstruction and to evaluate the correspondence between fragments using an optimistic estimator. Brown et al. [BTFN ${ }^{*} 08$ ] observe that in the case of degraded and eroded fracture surfaces, match uncertainty hampers feature-based matching; they propose a computationally efficient brute-force technique to exhaustively evaluate all possible least-squares alignments along edges of a set of fresco fragments (Fig. 8). A recent approach [AMK14] introduces a new method for pairwise matching of broken fragments from unorganized point clouds. The new descriptor contains both a cluster of feature points and curves along the principal directions of the cluster; point cluster is obtained by analysing micro curvature of the surface, while the associated curves are approximated using Fourier series. Matching function between curves is obtained by comparing the Fourier coefficients.

Other methods employ machine learning to directly learn match likelihoods from ground-truth matches, using feature vectors describing fragment coloration, texture parameters and surface markings $\left[\mathrm{TFBW}^{*} 10\right]$. Funkhouser et al. [FSTF*11] show that even when focussing on geometry alone but using a vast number of different geometric feature extractors (Fig. 9), supervised learning can significantly boost the discriminative power of a pair-wise match predictor, yielding well-calibrated probabilities whether a match is correct.

Many other works, too, combine geometric and texture/color information to disambiguate reconstruc- 


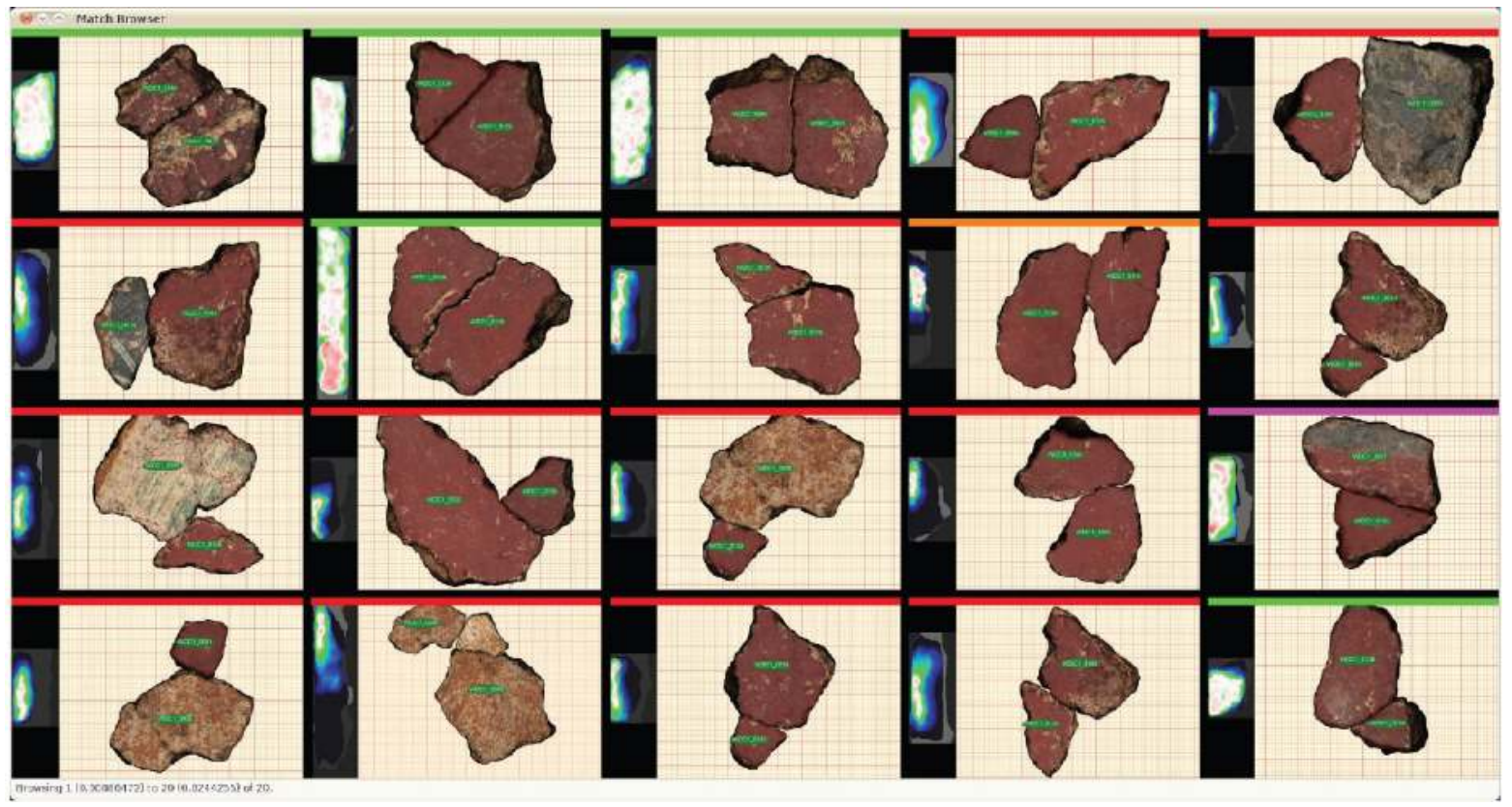

Figure 10: Match browsing tool. The match browsing application displays thumbnails of proposed matches along with a cross section of the matching area. The user marks correct matches (green bar), possible matches (orange bar), and incorrect matches (red bar). When the user confirms a match, all conflicting matches are marked with a purple bar. For instance, the 15th proposal above is impossible because it occupies the same edge of fragment WDC1_0124 as the second match, which has been confirmed correct $\left[B L D^{*} 12\right]$.

tion [WC08, KDSK10, ON11, ASC*13, ZL14]. Kleber et al. [KDSK10], for instance, use a shape matching algorithm based on features extracted from shape, marble texture, thickness of marble fragments, and location at the excavation, to reassemble the Ephesos marble plates.

Traditionally, the documentation, administration and scientific processing of archaeological fragments are carried out manually, an intensive and time-consuming effort. Hence, some researchers turn to investigating automatic systems and semi-automatic algorithms in the hope of expediting the reconstruction process and reducing the work from the archaeologists and restoration personnel. Kampel et al. [KM03] propose two reconstruction systems, adopting the traditional archaeological methodology of using the properties of the orientation and the profile of archaeological fragments. Based on such an approach, many works have tried to make the reconstruction algorithms either semiautomatic [MRS10,BLD*12] or fully automatic [GCBR*11, YWML11,PKT01,PK03,PAE* 12]. Belenguer et al. [BV12] put particular emphasis on implementation efficiency, speeding up geometric transformations, visibility tests and discretization operations by pre-calculating data and by moving all heavy calculations to the GPU.

While most reassembly approaches are bottom-up, starting from fragments, Shin et al. [SDF* 12] analyzed frac- ture line statistics of a (manually) assembled wallpainting to study the characteristic way that frescoes break (top-down). Some of the geometric features Funkhouser et al. [FSTF*11] incorporated in their match predictor were motivated by Shin et al.'s findings. Nevertheless, such reassembly by "inverse fracture" is only in its infancy.

Some useful tools have also been developed for assisting archaeologists to analyze and reconstruct archaeological objects from their fragments [LW04b, BLD*12, PPCS12]. Fig. 10 shows the semi-automatic matching tool proposed by Brown et al. [BLD*12]. Haptics can improve an assembly task by giving feedback when objects collide [RRC*10]; Olsson et al. [ONHC13] integrate a 6-degrees-of-freedom haptic capability within a fragment alignment tool for craniomaxillofacial surgery and archaeology applications.

\subsection{Discussion}

The majority of the works related to micro-geometric analysis focus on two main $\mathrm{CH}$ applications: restoration and perception enhancement (Fig. 11, right). In the first case, the local features are the key to reconstructing the (unknown) global shape of the final object; apart from the geometrical characterization at small scales of each single fragment, there usually is no other a priori information available to 
23

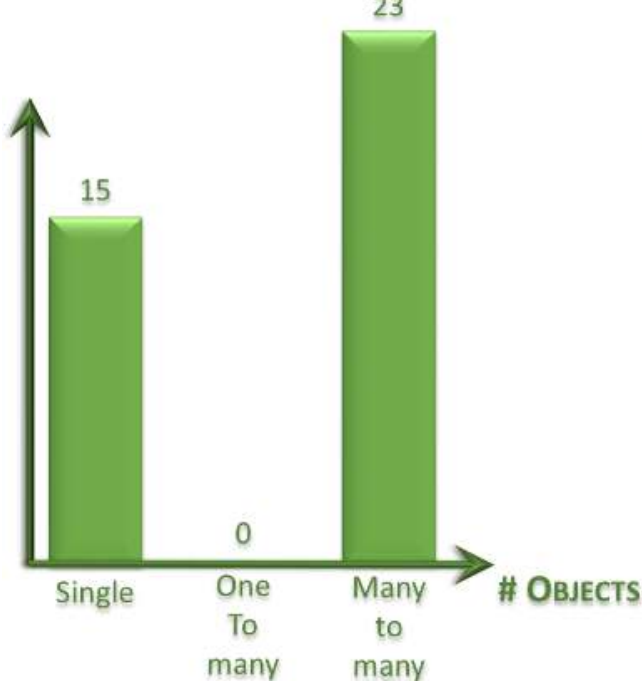

23

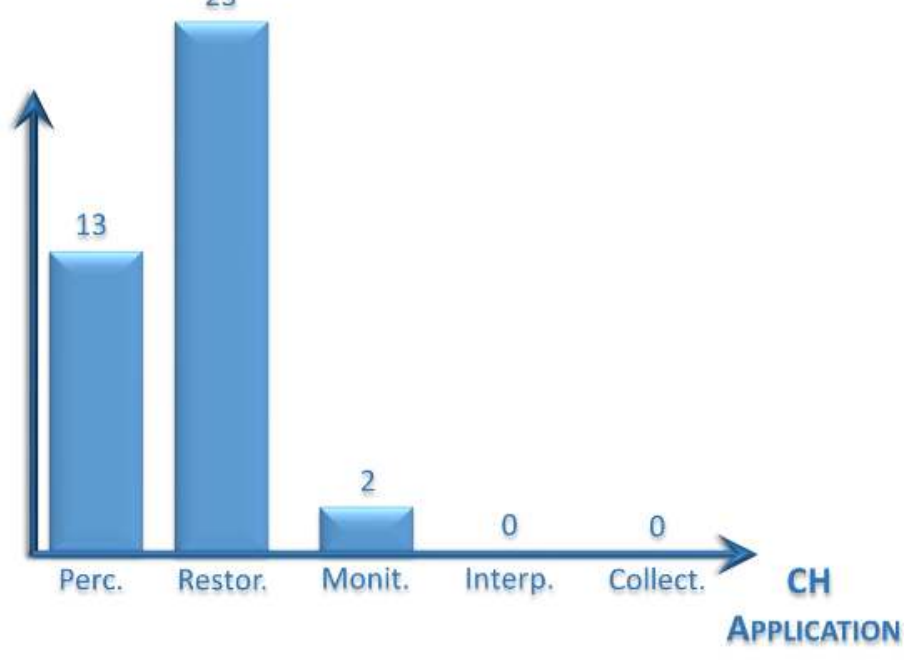

Figure 11: Micro-scale distribution. The majority of the works related to micro-geometric analysis focus on two main CH applications: restoration and perception enhancement. All the restoration approaches are of course classified as many-to-many, while almost all single object analysis belongs to the perception enhancement class.

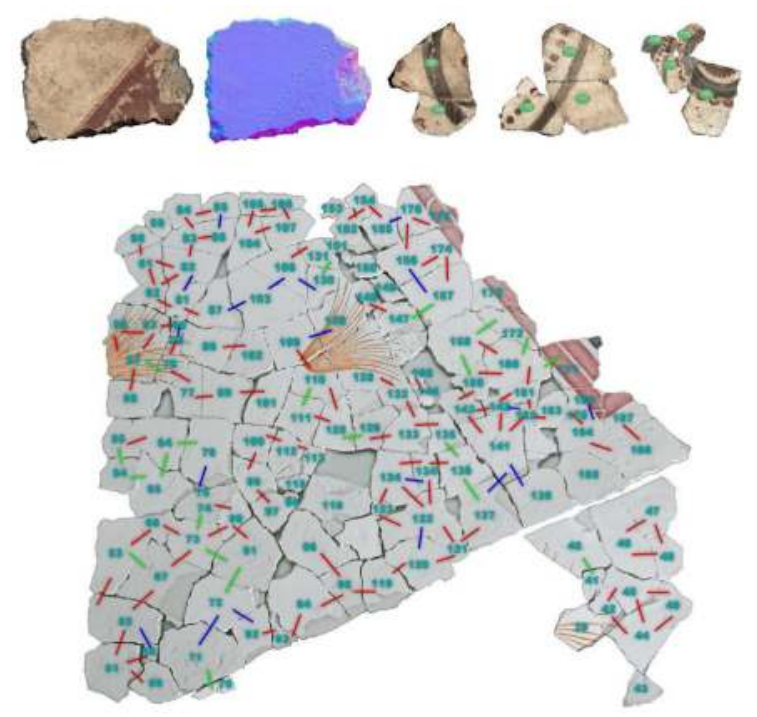

Figure 8: Reassembling fresco fragments. Top row, from left to right: apart from small scale $3 D$ features, acquired colors and high-resolution computed normals are important cues for reassembling purposes; the last three images are examples of possible matches between fragments. The bottom row shows the entire "ground truth" synthetic fresco. Red, blue and green lines indicate the matches found by the algorithm. The numbers indicate the fragments that have been $3 D$ scanned [BTFN* 08$]$. solve the reassembly problem. The second case aims at improving the perception of small-scale details of the 3D model of a given artifact, which is a valuable tool to assist scholars in their research work. In contrast, the works classified in the monitoring, interpretation and collection analysis groups use different pipelines and mathematical representations depending on the $\mathrm{CH}$ item involved; they require specific information that is not independent from the particular kind of object, and is typically visible at meso or macro scales. The single work we have found in the monitoring group proposes a general framework, where the differences over time are obtained using micro features, and it can be applied to a wide range of objects, regardless of their global or local behaviour.

All the restoration approaches are of course classified as many-to-many, since they exploit the combinatorial comparison between features from different pieces being reassembled. Conversely, almost all single object analysis belongs to the perception enhancement class. This is because the operator to increase detail readability has been independently applied to a single $\mathrm{CH}$ sample, without taking into account any other information coming from similar items.

\section{Meso geometry}

In this section we organize the literature related to the techniques that studies local parts of a 3D model to perform analysis in the $\mathrm{CH}$ field. 

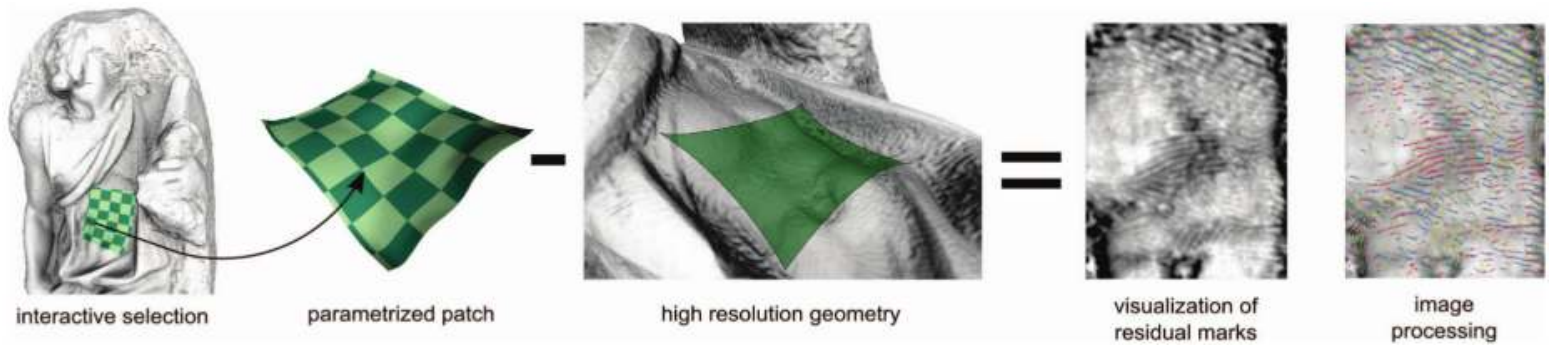

Figure 12: Residual marks detection. A perception enhancement pipeline based on a local flattening of a portion of a $3 D$ model, followed by an image processing step applied to the resulting $2.5 \mathrm{D}$ map. The user manually selects a portion of the $3 D$ model, the algorithm finds a parameterization on the patch, and a representation of residual marks is obtained. Finally, image processing allows the user to classify different chisel marks. Courtesy of Pietroni et al. [PMCS11].

\subsection{Single object analysis}

Perception enhancement. Pietroni et al. [PMCS11] present a pipeline to analyze the $2.5 \mathrm{D}$ information distributed over $3 \mathrm{D}$ geometry, using a local 2D parameterization. They show the effectiveness of their method to solve specific $\mathrm{CH}$ tasks, such as the study of chisel marks over the surface for tool identification (Fig. 12). Schmidt et al. [SSB11] transform a $3 \mathrm{D}$ model into a $2.5 \mathrm{D}$ representation as well, and apply image-based processing to the depth and reflectance signal to extract lines and contours in Chinese Buddhist stone inscriptions.

In a similar vein, Pal et al. [PTW13b, PTW13a, PSP*14] present an interactive application for browsing severely damaged documents and other cultural artefacts, which contain distortions such as wrinkling, buckling, and shrinking. In contrast to the above, their goal is not only to study the $2.5 \mathrm{D}$ structure, but to flatten local regions of the document for better readability of the original text, which would be impossible to do for the physical items due to their fragile condition (Fig. 13). Their system consists of an interactive viewer that allows a user to browse a document while dynamically flattening only the local region under inspection. A follow-up work [PSP*14] exploits the flattening for a global restoration of damaged documents (see section 6.1).

Restoration and preservation. In the field of document restoration, Pintus et al. [PMW* 09] present a technique that uses a flatbed scanner and Photometric Stereo (PS) [Woo79] to identify and repair scratches or tears in old document or photographs (Fig. 14). They exploit normal estimation to find creases in the almost flat surface, and they apply an infilling operator to restore original pixels. Although the texture synthesis is trained with the information from the entire image (i.e., the non-damaged part), the restoration is performed locally and it is driven by the content of the image in the local neighborhood of the damaged pixel. Conversely, in section 6.1, document restoration is a global transformation (typically an undistortion) applied to the whole page.

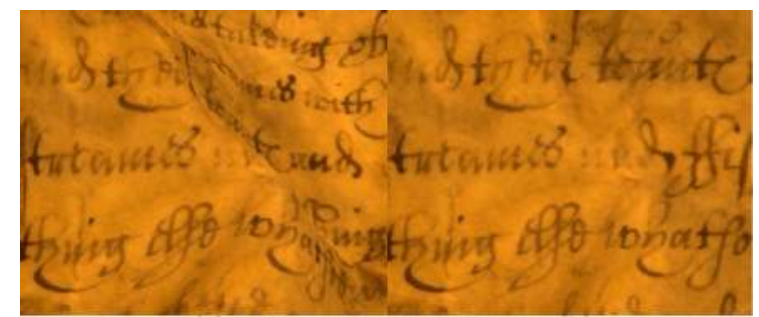

(a)

(b)

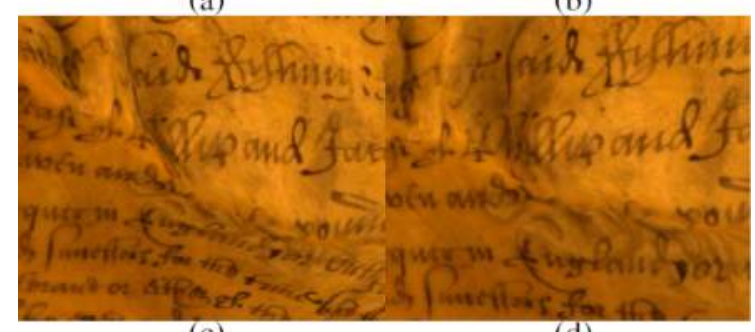

(c)

(d)

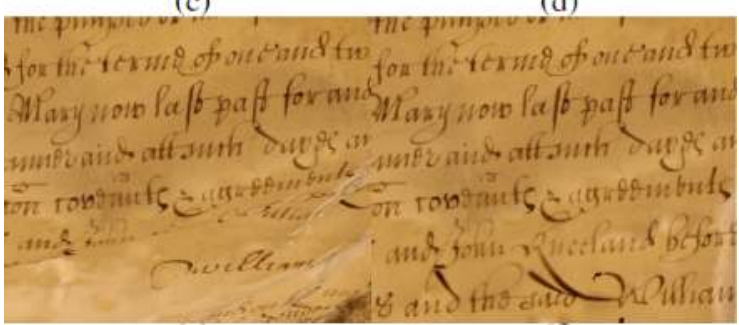

(e)

(f)

Figure 13: Interactive browsing of damaged documents. Severely damaged documents are acquired in $3 D$ using a Structure-from-Motion approach. A dynamic flattening is performed while the user browses the document in order to increase its readability. We show sections of the mesh rendered in local-affine mode without flattening (left) and with local-flattening mode (right). Note that the original wrinkles remain visible since the shading is baked into the texture [PTW13b]. 

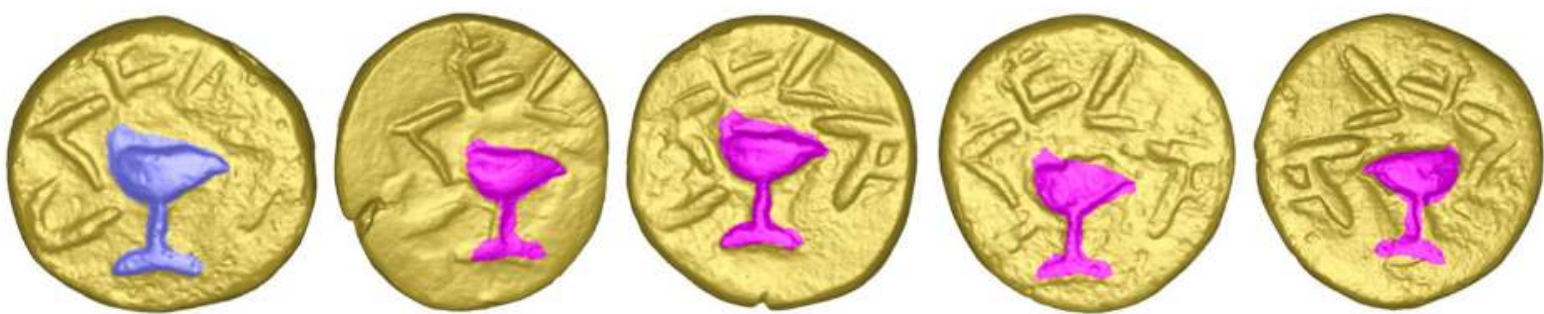

Figure 17: Shape retrieval of coins. The algorithm finds the magenta goblets given the blue query. The coins are different in terms of the goblet shape, its location, level of noise, and many other factors. Courtesy of Tal et al. [IT11].
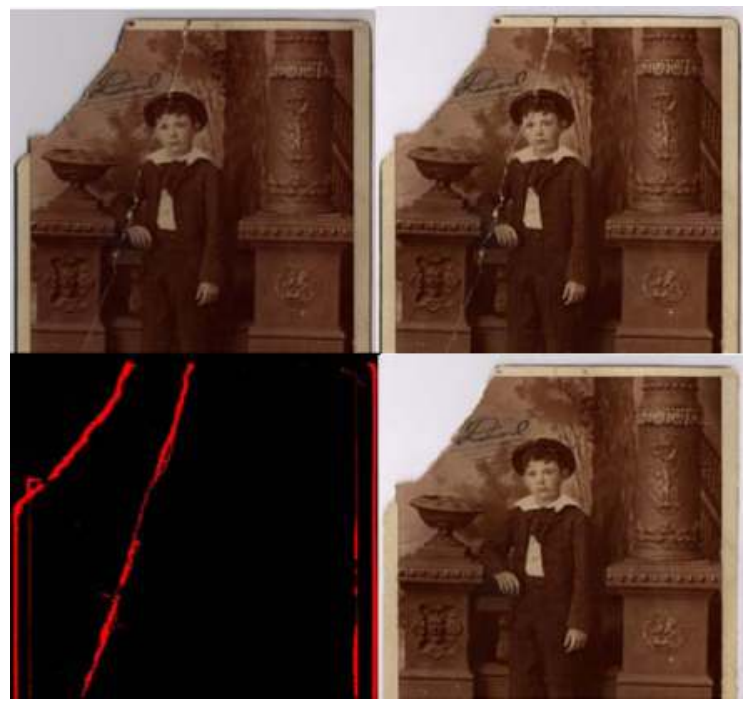

Figure 14: Document restoration. First row: two images acquired with a flatbed scanner that incorporates two separate and independently controlled illumination bulbs. By using photometric stereo [Woo79] tears and creases are extracted (low-left), and infilling algorithms are applied to digitally restore the image (low-right) [PMW* 09].

\subsection{One-to-many analysis}

Collection analysis. There is an increasing demand within the humanities and social sciences to use digital databases for rapid queries and fast browsing of huge sets of artworks. In this scenario, analysis based on meso geometry plays an important role, since it enables mathematical representations of such items in terms of local geometric feature vectors. These give a numerical description of sub regions of an object for its classification and recognition. 3D scanning technology is a standard archaeological tool for pottery analysis. Koutsoudis et al. [KPC10] developed a method to detect similarities in 3D pottery repositories. They designed two shape descriptors (Fig. 15). The first is based on the axial symmetry of common potteries, and consists of a set
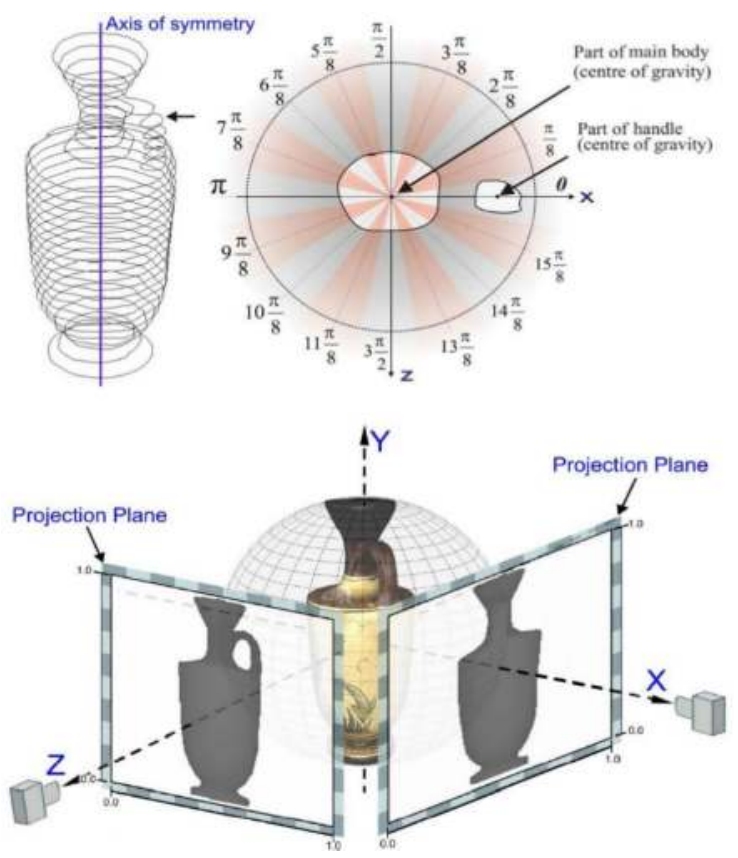

Figure 15: Pottery shape descriptors. Top: set of curves by intersecting the pottery and a plane orthogonal to its axis of symmetry. Bottom: set of two depth maps by projecting the object onto two orthogonal planes. Courtesy of Koutsoudis et al. [KPC10].

of curves obtained by intersecting the pottery surface and a plane orthogonal to its axis of symmetry; the other is a set of two depth maps obtained by projecting the object onto two orthogonal planes, which are both parallel to the vessel's axis of symmetry. They employed these descriptors into a web-based 3D pottery search engine (Fig. 16). Karasik et al. [KS08] acquired about a thousand of potsherds with variable shapes, sizes and surface properties from several sites and periods, and exploit this large range of archaeologically relevant pottery types to test an algorithm to extract their overall axis of symmetry and their mean profiles. They analyze the particular morphology of pottery [KS11] by using 


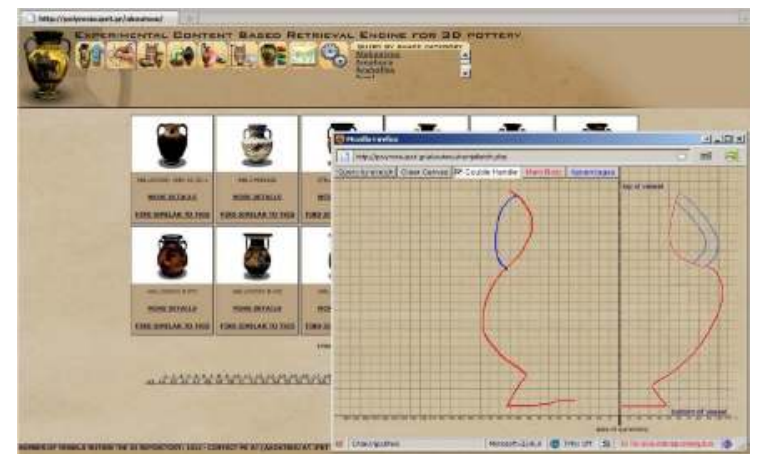

Figure 16: 3D Pottery Search Engine. Web-based 3D application to perform query-by-sketch. Courtesy of Koutsoudis et al. [KPC10].

three mathematical representations of the pottery items' profiles, and defining a proper metric to measure the distance between them. Cluster Analysis and Discriminant Analysis are then used to reveal a hierarchical classification of possible assemblages; a Procrustes analysis is also used when dealing with a global comparison between artifacts (see section 6.2). Finally, the Pottery Informatics Query Database (PIQD) [SKN*12] is another example of quantitative analysis applied to large sets of cultural heritage items. This is an open-source online tool that enables scholars and students to test humanities-related hypotheses against ceramic data, by defining a way to build comprehensive ceramic taxonomy and their regional spatial patterning.

Itskovich et al. [IT11] used a surface partial-matching technique in a shape classification and retrieval scenario; they apply the method to Hellenistic vases, lamps and coins inside a database, given a portion of the object as a query geometry. Their algorithm is capable of matching the correct item, even if they differ in the query location within the surface and in the level of noise.

\subsection{Many-to-many analysis}

Restoration and preservation. Contrary to pure feature based frameworks (see section 4.2), some assembly processes incorporate a priori knowledge about the application domain. We classify them as meso-geometric analyses, because they extract local descriptors, but they are not independent on the local or global object geometry. Pottery reconstruction, for example, benefits particularly well from exploiting the fact that most pottery was created on a potter's wheel, that is, the final assembly is expected to be radially symmetric [WC04a, WC04b, WC08] (Fig. 18(a)). In its most stringent form, however, this assumption may prove unstable in the presence of very small or flat fragments [KS04] and wherever there are larger imperfections in the earthenware. Cohen et al. [CLE12] hence choose less-stringent continuity criteria by matching weighted curve moments across adjacent sherd surfaces (Fig. 18(b)), and Kikuchi et al. [KO13] pursue a similar goal in the context of cranial reconstruction from skull fragments: they fit a parametric Bézier surface to the surface of each neurocranial fragment to predict the surface shape of adjacent fragments, thus finding matches through surface extrapolation rather than just contour matching. Similar approaches exploit skull symmetry to drive the re-assembly and completion tasks [LYW*11, WYLL11]. Other applications, such as architectural reconstruction from fractured masonry, require extracting different structural cues, such as the main orientation of architectural features. A particularly challenging case are Gothic spires, where Laugerotte et al. [LW04a] extract dominant directions in a way that is resilient to the presence of ornamental features. Although lost, when the overall shape of a destroyed monument is known roughly, virtual anastylosis [CFB* $\left.13, \mathrm{TFK}^{*} 09\right]$ is another application that performs local geometrical analysis for the archaeological and architectural reconstruction of a historic site, after a careful study of the remaining original elements.

\subsection{Discussion}

Meso-scale methods in the perception enhancement typically deal with local surface flattening, while those in the restoration class use the same pipeline as in the micro-scale based works, but also exploit the knowledge of the object topology. The majority of them combine micro feature extraction with the analysis of a bigger neighborhood. For instance, the possibility to locally represent the surface as a plane enables the flattening strategy, or the analysis performed on vessels benefits significantly from their cylindrical structure. However, compared to other techniques, the meso-scale analysis is the best choice for collection analysis applications. The extraction of features that depend on local geometry enables the studying of a database with a huge number of object with the same topology but with a high rate of variability. The analysis at this scale produces a good classification and successful retrieval in many practical $\mathrm{CH}$ cases, such as pottery, vessels, lamps, and coins. Compared with the micro-scale analysis, the distribution of the methods in the single and many-to-many classes is very similar, with almost all the restoration approaches in the many-tomany class, while the perception enhancement in the single group. However, in addition we have here some techniques in the one-to-many class, all of them related to collection analysis.

\section{Macro geometry}

In this section we organize the literature related to the techniques that studies the entire shape of a 3D model to perform analysis in the $\mathrm{CH}$ field.

Typical 3D models are obtained with triangulation laser 

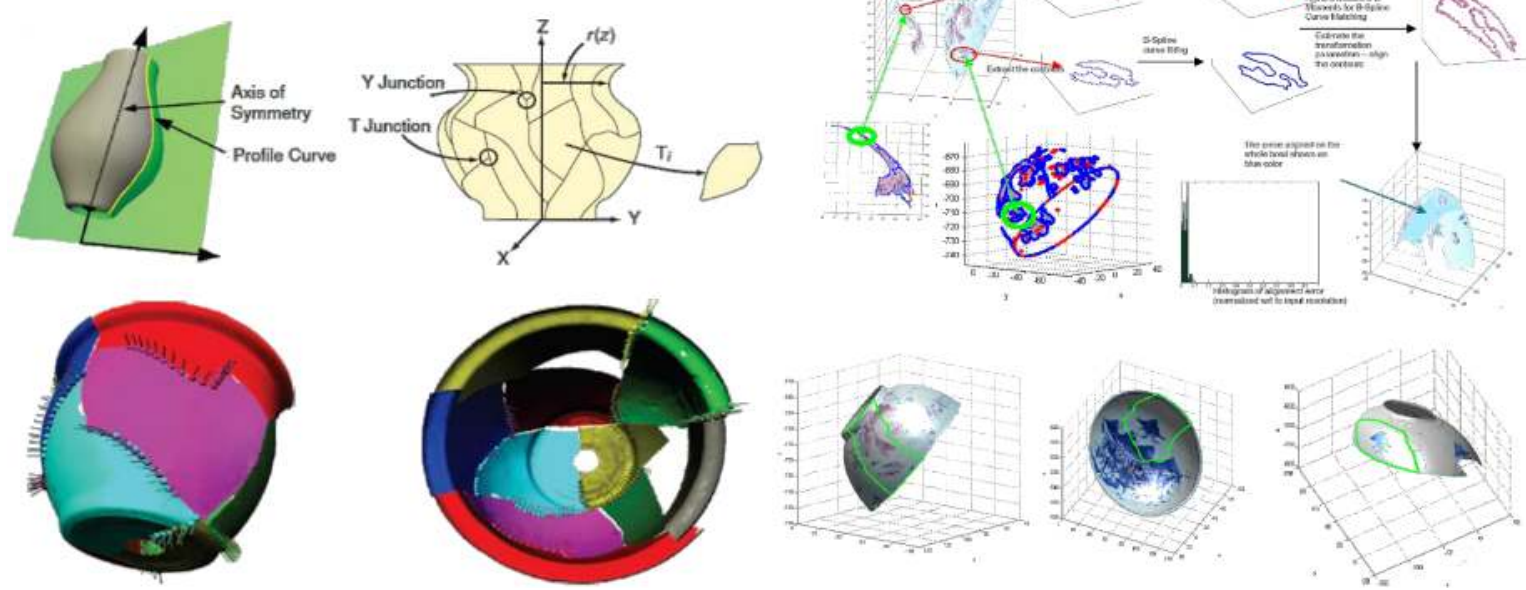

(b)

Figure 18: Reassembly by meso-scale features. (a) radial symmetry constraint (courtesy of Willis et al. [WC08]); (b) matching by weighted curve moments across adjacent sherd surfaces (courtesy of Cohen et al. [CLE12]).
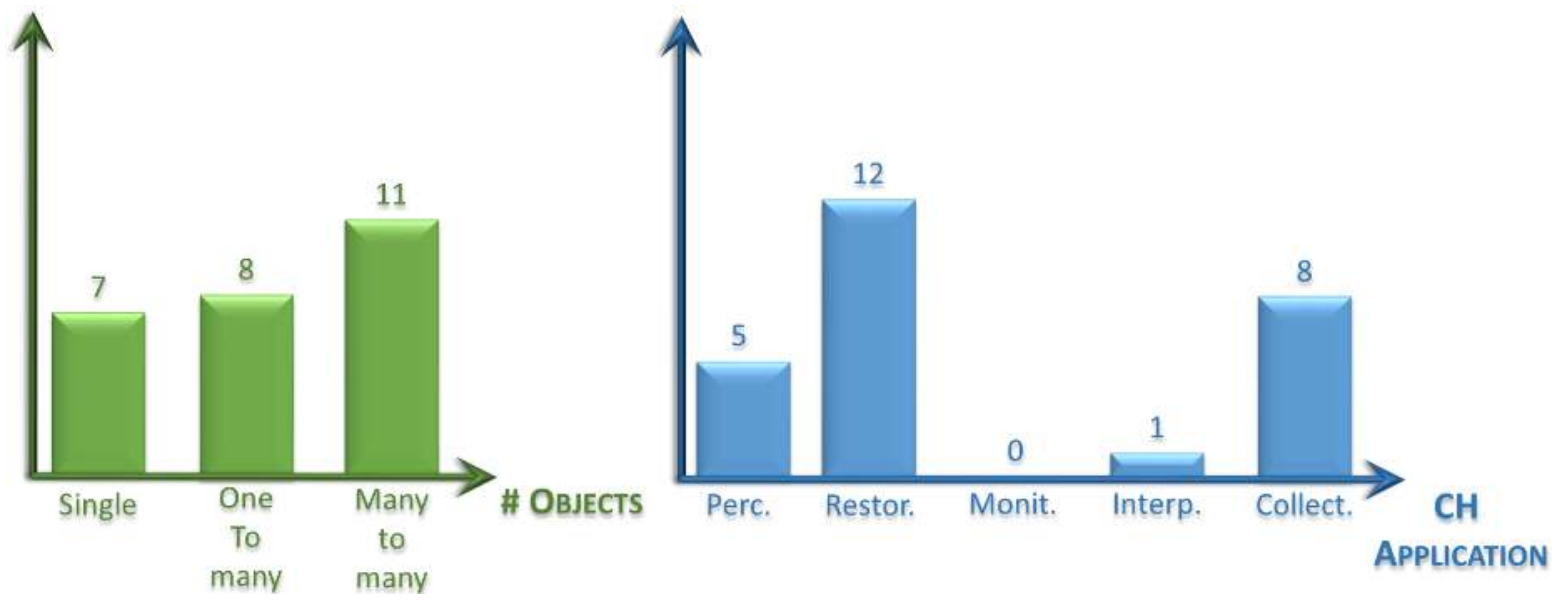

Figure 19: Meso-scale distribution. Methods in the perception enhancement deal with local surface flattening, while most of those in the restoration class are related to reassembly fragmented objects. The restoration approaches are classified as manyto-many, while almost all single object analysis belongs to the perception enhancement class. We have here some techniques in the one-to-many class, all of them related to collection analysis.

scanning, time-of-flight scanners or CT volumetric acquisition devices.

\subsection{Single object analysis}

Perception enhancement. A useful way to document archaeological finds is the representation of so-called rollouts. The analysis of rotation-symmetric objects with paintings or inscription benefits from rollouts because they give an overall view of the $\mathrm{CH}$ content [RMK13]. Typically they are created either by manual drawing or from photographs. Con- versely, generating $2.5 \mathrm{D}$ rollouts from color and geometry acquisitions represents a more reliable framework, which assists the scholars in the iconographic interpretation task. Pitzalis et al. [PCMA08] apply this analysis to the "Cylinder seal of Ibni-Sharrum", a finely engraved object; its rollout reveals in one single $2.5 \mathrm{D}$ basrelief the entire structure of the fine drawings in the seal (Fig. 20). They combine this pipeline with the detail enhancement technique proposed by Cignoni et al. [CGPS08] (see section 4.1). Bechtold et al. [BKMK10] combine the geometry and the color to produce rollouts of figurative vase painting. They use 3D geom- 

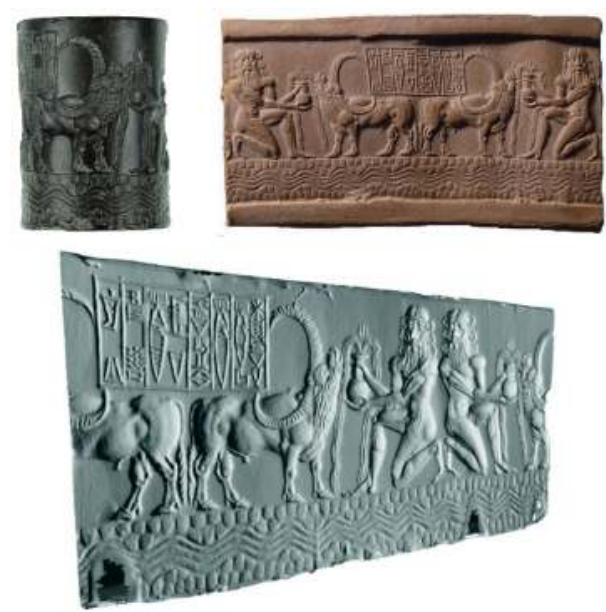

Figure 20: Increasing perception with rollouts. Top: the "Cylinder seal of Ibni-Sharrum" and its development on clay; Bottom: the result of an unwrapping of the cylinder followed by an inversion of the depth (negative rollout). Courtesy of Pitzalis et al. [PCMA08].

etry from a structured light scanner to drive the transformation of the vase cylindrical topology into a $2.5 \mathrm{D}$ surface. For study by handling replicas, one of the most important issues is the perception and readability of the details in the printed replica (see section 4.1). Laycock et al. [LBM*13] used xray micro- CT technology to scan one of the 19th Century Cantonese Chess pieces with a voxel resolution of less than $9 \mu \mathrm{m}$. They use a straightforward scaling of the entire model, by $3 \mathrm{D}$ printing the model at a higher scale; this enables the scholar to appreciate both the overall structure of the chess piece and the fine surface details imperceivable in the original. Further, since those objects have an intricate internal structure, they virtually dismantle each part of the piece, so that they allow scholars to study those items separately.

Restoration and preservation. Document analysis is a topic of broad scope. Here we consider techniques that use advanced representations (such as $2.5 \mathrm{D}$ geometry and surface normals) to virtually restore documents and analyze their content. Documents can degrade in numerous different ways as they age. Much work has been carried out on virtually correcting the geometric distortions, scratches or tears. Some of the existing methods focus on solving the problem locally (see section 5.1), while others take into account the entire page to produce more physically coeherent restoration. These latter approaches correct large distortions by capturing 3D triangle meshes of the document shape and flattening them using geometry processing techniques [BS04, SYY*05, BSY*07, PSP*14]. Others correct finer geometric distortions using image-based methods [LSC08,Lan13]. Lin et al. [LLS06] present a framework to preserve $3 \mathrm{D}$ documents, such as fragile bound manuscript

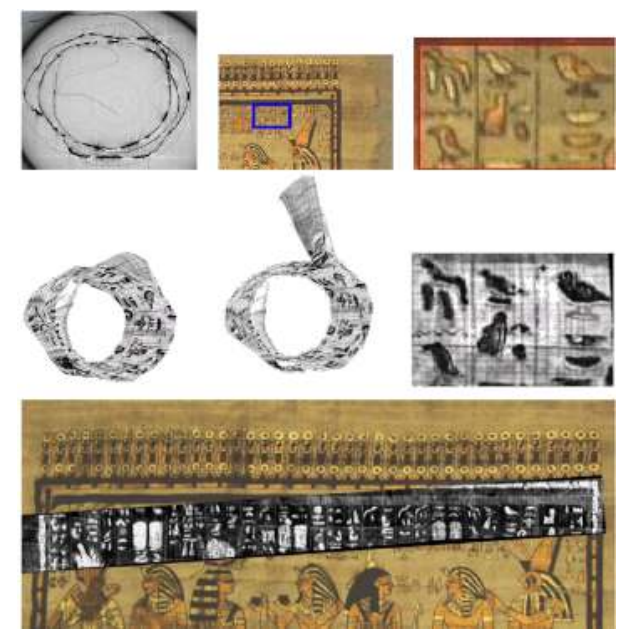

Figure 22: Volumetric scanning of a rolled papyrus. Top: one slice of a CT-scan, a digital image of the origianl item, and a close-up view. Center: two views of the particle system simulation, and a detailed view after unrolling. Bottom: a comparative evaluation by overlapping the unrolled information from CT-scan and the original papyrus. Courtesy of Seales et al. [SLO4].
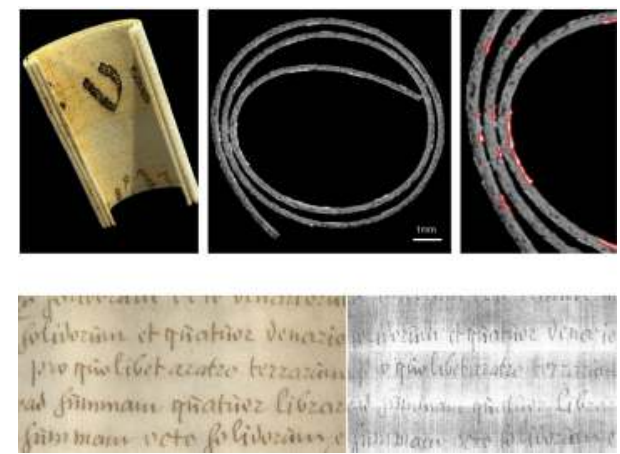

Figure 23: $X$-ray micro-tomography applied to restoration. Top: a small region of a rolled historical parchment acquired with a X-ray micro-tomography scanner. We show from left to right the rendering of a cut of the volume with pseudocolor, a tomographic slice, and a close-up view of it with highlights in the ink regions (red). Bottom: the comparison between the original unrolled parchment and the reconstruction obtained by virtually unrolling the X-ray volumetric data of the scrolled parchment. Courtesy of Samko et al. [SLMR14].

with a non-uniform surface, documents engraved on stone, or scrolls that have been entombed in volcanic ash. They use $3 \mathrm{D}$ technologies to acquire the surface and to produce a physically-based 3D-to-2D transformation that converts the $3 \mathrm{D}$ model to a $2 \mathrm{D}$ image, subsequently preserving and giv- 


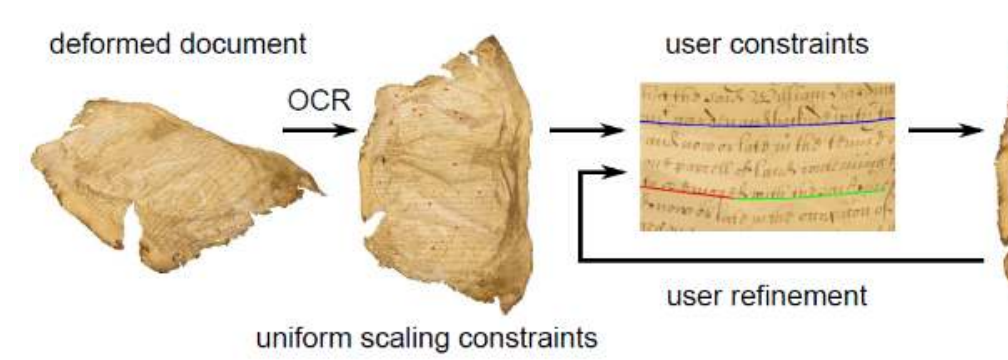

uniform scaling constraints

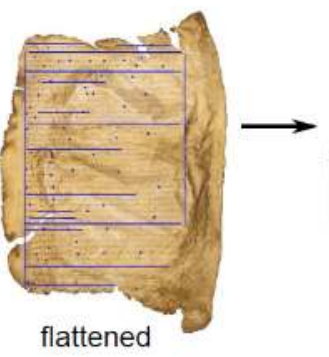

flattened

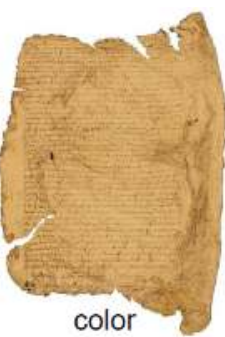

corrected

Figure 21: Interactive restoration of historical documents. All steps of the restoration algorithm presented by Pal et al. [PSP* 14] and applied to historical documents. From left to right: the original distorted document is flattened using the Jacobian estimated from the OCR analysis. An interactive refinement of the constraints improves the restoration, and is iterated until the desiderata of the domain expert are satisfied. Ultimately, we remove the intensity and color variations from the texture $\left[P S P^{*} 14\right]$.

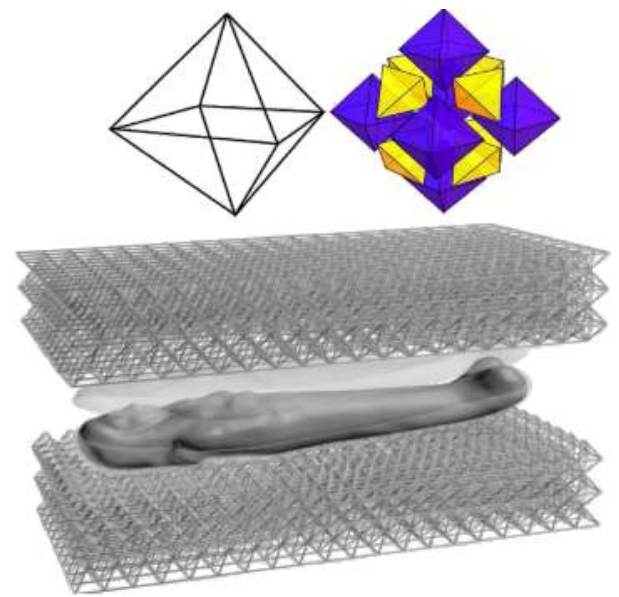

Figure 24: Geometric analysis for packaging applications. Top: the octahedron, which is the basic unit that has been used to build the $3 D$ printed package. Bottom: The final structure ready to print. Courtesy of Sá et al. [SEG*12].

ing a restitution of its intrinsic textual information. Another non-destructive method [SL04] was presented to reveal inaccessible text buried within damaged books and scrolls. This method is based on volumetric scanning, data modeling and physically-based simulation; it recovers readable text without physically opening or damaging the fragile items (Fig. 22). Samko et al. [SLMR14] use X-ray microtomography to acquire a volumetric reconstruction of a scroll of parchment that, due to its state of preservation, cannot be unrolled. The X-ray imaging picks up ink concentration, allowing for 3D reconstruction of the ink distribution followed by virtual unrolling of the scroll's content (Fig. 23).

Extensive and global morphological study of 3D surface characterization represents another invaluable source of qualitative and quantitative information to predict the proper restoration procedures. An important example is the scientific investigation employed in the restoration of Michelangelo's David [CCG*04], which produced guidelines for integrating 3D data analysis in cultural heritage applications. They perform physical measures as the height of the David, its surface area and the volume; they also analyze the global geometry of the statue to evaluate the exposure to falling contaminants, such as rain, mist, or dust. Finally, a study is performed to visualize the center of mass projection onto its base; this helps to understand how the weight of the entire statue is distributed, and which are the corresponding weakest parts that must be preserved and eventually renovated. The high-resolution 3D model of the "Pietà" [BRM*02] has been used by Wassermann et al. $\left[\mathrm{WCV}^{*} 03\right]$ to study the statue's damage and subsequent repair.

Moreover, the analysis of captured 3D models and the use of rapid prototyping are crucial for conservation and $\mathrm{CH}$ restitution as well. Although extensively used for perception enhancement (see section 4.1), 3D printing plays an important role in the preservation field. Transporting and storing the artifacts are complex issues, involving different packaging solutions, such as double boxing, padding negative spaces and cushioning braces. A recent paper [SEG* 12] proposes the use of the generative modeling language in order to analyze the $3 \mathrm{D}$ model of artworks and to $3 \mathrm{D}$ print unique 3D-fitted containers for packaging them (Fig. 24).

Some methods cope with the virtual and physical reconstruction of missing part of a badly damaged $\mathrm{CH}$ items [FMOS11]. They try to perform a completion of those parts by using the information from the scans of the whole ruined sample (e.g., by exploiting symmetry of certain objects), or from other possible hypotheses of structures based on drawings, descriptions and old texts. They also analyze feasibility of potential structural configurations [LD06]. Hess et al. [HRM $\left.{ }^{*} 09\right]$ combine 3D laser scanning and anthropological research to deliver a holistic virtual 3D reconstruction and multimedia interactive experience of a Melane- 
sian war canoe. The combination of reverse engineering and rapid prototyping offers a tool that overcomes limitations in some completion frameworks. Completion of the skeletal material $\left[\mathrm{FdCP}^{*} 08\right]$ is crucial due to the fragility of the skeletal findings, which should limit the over-handling of these fossils, and the rarity of these remains, which precludes any task that assembles together existing and reconstructed fragments. Hence, digital modeling, 3D scanning and rapid prototyping together are essential tools that can be usefully applied for reversible and non-invasive restoration processes of damaged bones, by providing a virtual and physical restitution of a whole bone and preserving the original one.

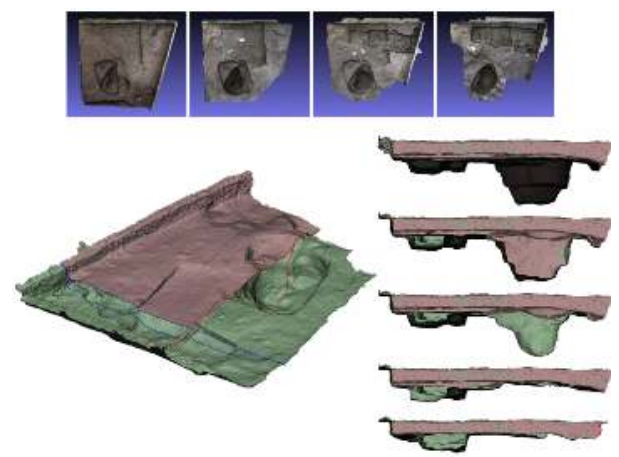

Figure 25: Monitoring the excavation with photogrammetric approaches. Top: acquired $3 D$ model of the same archaeological site in different times, showing the evolution of the excavation. Bottom: interactive slicing visualization to highlight the evolution of the excavation in specific areas of the site. Courtesy of Dellepiane et al. [DDUC $\left.{ }^{*} 13\right]$.

Monitoring. Geometric acquisition and measurements produce snapshots that can be used to track global conditions of an object through time, for instance to document the evolution of an archeological destructive intervention, or based on the idea that changes over time might indicate damages. Archaeological sites are problematic case studies in the monitoring field, both during their excavation and for their longterm preservation (see also section 4.1). In the former case, fast $3 \mathrm{D}$ acquisition techniques are required, since a complete geometrical capture of the working site is required every day, or even every few hours. Automatic dense stereo reconstruction tools and 3D scanning are particularly suitable for monitoring of excavations [DDUC ${ }^{*} 13, \mathrm{CDD}^{*} 11, \mathrm{DRDSH}^{*} 14$, $\left.\mathrm{KOC}^{*} 14\right]$, and, coupled with the proper interactive tool (e.g., Meshlab [RCD*12] or Hyper3d [KRF*14]), they provide a useful workflow for objective spatio-temporal analysis and documentation (Fig. 25).

In civil engineering, recent work on the computational and mechanical analysis for evaluating the structure and stability of bridges integrates the results of close-range digital photogrammetry, CAD tools and finite elements analysis (FEM) [AADC* 07, RCAS11, RSdA*13]. Close-range pho- togrammetry has also been used to locate structural problems in cultural heritage monuments [AHLO05]. Some techniques analyze the damage and deformation which may have occurred depending on different aspects of construction [ARPLA10,SVG09]; they can also allow engineers and architects to estimate the influence of geometric variations in the distribution of stress [ACCLA07]. Finally, De Balestrini et al. [FdBBB*13] presented a survey methodology to investigate earthquake damages of the "Camera degli Sposi" of Mantegna (Mantova, Italy). They use both time-of-flight and triangulation laser scanners to produce multiresolution morphological maps of deformation, that highlight the damaged parts of the building and help to understand the damage mechanisms.

The result of the diagnostic phase is a mandatory prerequisite for the evaluation of any kind of intervention or restoration in any architectural, or artistic framework. The incomplete nature of petroglyph drawings and photographic data makes them insufficient to track the effects of natural destructive processes; $3 \mathrm{D}$ laser scanning was found useful to record and monitor the erosion of rock art [BCDA*05].
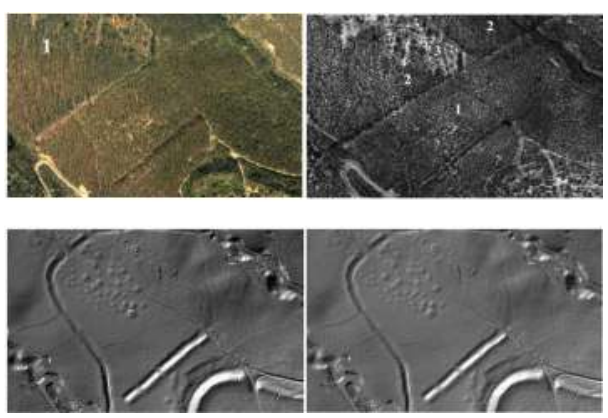

Figure 26: Analysis of airborne laser scanning data for archeological sites identification. Top: aerial and intensity image of the studied area. Bottom: resulting DTM after processing, which shows possible archaeological sites once the information of forested areas has been removed. Courtesy of Doneus et al. [DB06].

Interpretation. An important attribution problem in $\mathrm{CH}$ consists in the identification of structures that are manmade within cluttered environments. Recent studies have examined the possibility of using LiDAR in archaeological investigations to map and characterize earthworks, to capture features that may be indistinguishable on the ground and to aid the planning of archaeological excavation campaigns [LCM11, DACC05]. The approach proposed by Doneus and Briese [DB06] aims at analyzing airborne laser scanning data to identify archaeological sites within forested areas Fig. 26. It relies on the capability of such an acquisition device of penetrating forest to a certain degree (depending on the vegetation density), and on the possibility to compute the terrain surface even in wooded areas. In this way they are able to distinguish natural and recent features, like 
brushwood, or piles of twigs or wood, with small features related to the presence of an archaeological site.

Recently, high interest has arisen in the 3D morphological analysis applied to interpretation of works of art. Dellepiane et al. [DCF* 07$]$ use the data from 3D scanning and image-to3D-model mapping in order to measure the plausibility of an attribution hypothesis of a Renaissance artifact. In a metrically objective framework they compare the global shape of a digital model of a small bronze horse to a silverpoint drawing by Leonardo, which qualitatively displays a high degree of similarity, and was thought to be drawn by directly looking at the bronze. Using image-to-3D-model mapping techniques, they were able to superimpose the 3D model with the Leonardo's drawing, giving scholars a better objective cue to solve and assess the issue of that particular disputed hypothesis. Muzzupappa et al. [MGM*12] address the interpretation problem related to the "Riace Bronzes" statues. In particular they exploit 3D scanning techniques to study the right hand of the "Bronzo A". They provide archaeologists with some objective data about the hand shape, by measuring a series of geometrical data such as cross-sections, deformations and volumes. This approach helps to enforce the hypothesis about the object in the hand, which is one of the fundamental steps to understand the statue's identity. Nadel et al. [NFRM15] use a combination of multi-view reconstruction, 3D macro feature extraction and geometrical analysis to address archaeological study of prehistoric bedrocks, and the corresponding raised questions concerning their chronology, the techniques used to make them, how their forms and functions are correlated, the spatial distribution of these works of art across different sites as well as their social and cultural contexts. For each of them they extract a dense 3D model, with a millimeter resolution, and they characterized it by shape measurements such as volume, sections, and vertical and horizontal reflective symmetries. The use of 3D modelling and rapid prototyping is employed by Docchio et al. [DST05] in order to explore and verify a new hypothesis on the origin of the "Vittoria Alata" bronze statue, by performing a set of measurements related to distances between pairs of fiduciary points suggested by archaeologists.

In addition to attribution and identification, geometric analysis is used for the investigation of the motivation for a work or its alteration. A high resolution 3D model of Michelangelo's Florence Pietà [BRM $\left.{ }^{*} 02\right]$ was used by Wasserman et al. [WCV*03] to analyze Michelangelo's motivation for breaking apart the statue which was subsequently reassembled by another artist. By virtually removing pieces of the work that had been repaired, Wasserman et al. were able to present the piece post-damage, and observe that it is similar in form to subsequent work by Michelangelo. They claim that the Michelangelo in breaking off pieces of the statute was acting to produce another sculpture, rather than in a fit of anger.

\subsection{One-to-many analysis}

Restoration and preservation. Given a damaged face appearing in a 2D image (e.g., from paintings), Lanitis et al. [LS09] describe a method to perform virtual restoration. They estimate the complete 2.5D shape of a face using data from the non-damaged face. Then, they compare the face with other similar samples in the training set of nondamaged faces, and predict the texture of the damaged regions. They use a similar texture synthesis approach as in Pintus et al. [PMW*09] (see section 5.1), but in their restoration framework they exploit the global transformation between the $3 \mathrm{D}$ and the $2 \mathrm{D}$ domain, by mapping the restored geometry and color back onto the original image.

Monitoring. Methods that allow to compare multiple different datasets (floor plans, drawing, 3D scans) and to explore possibilities about construction and layout are employed by Fiorillo et al. [FRB ${ }^{*} 13$ ] to monitor the historical representations of a site over time. They compare the accurated 3D model obtained from a laser scanner system with ancient manual drawings. The basic idea is to check the global dimensional accuracy and the overall formal correctness of surveys performed by three different authors in the late $18^{\text {th }}$ and in the $19^{\text {th }}$ centuries.

Collection analysis. 3D scanning campaigns allow for an extensive study on how to compare similar digital objects within massive 3D databases [GCJ*07]. For instance, recently, hundreds of Gallo-Roman white clay figurines coming from the center of France have been digitized using 3D scanners. Bourdeu and Pitzalis [BP10] presented a method to quantify and compare the shapes of those models. After placing homologous points (landmarks) along the entire object surface, they use geometric morphometrics, and in particular the Procrustes analysis, to extract an estimation of the difference between two shapes. This framework assists the scholars in determining the provenance of the figurines based on an objective estimation of statistical shape variability.

\subsection{Many-to-many analysis}

Restoration and preservation. Lu et al. [LZT*11] use the analysis and quantitative comparison between different $3 \mathrm{D}$ digital models of statues in a many-to-many framework, in order to solve the problem of restoring the facial sculptures in the city of Bayon. After performing a rough shape recovery using all parameterized samples in the scanned data, they performed hierarchical clustering to group similar faces. Then they refined the restoration applying the shape recovery algorithm to high resolution data in each respective cluster (Fig. 27).

Collection Analysis. Shape comparison by using digital copies draws increasing attention in modern culture heritage studies [ZLZ*13]. Recently, a technique is presented [LZZ*13] that analyzes portrait sculptures of Augus- 


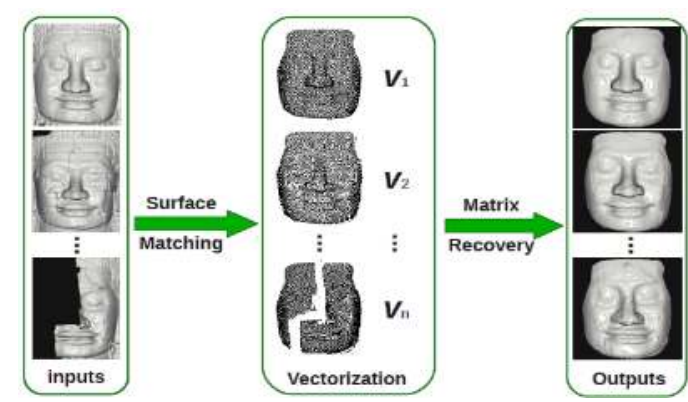

(a)
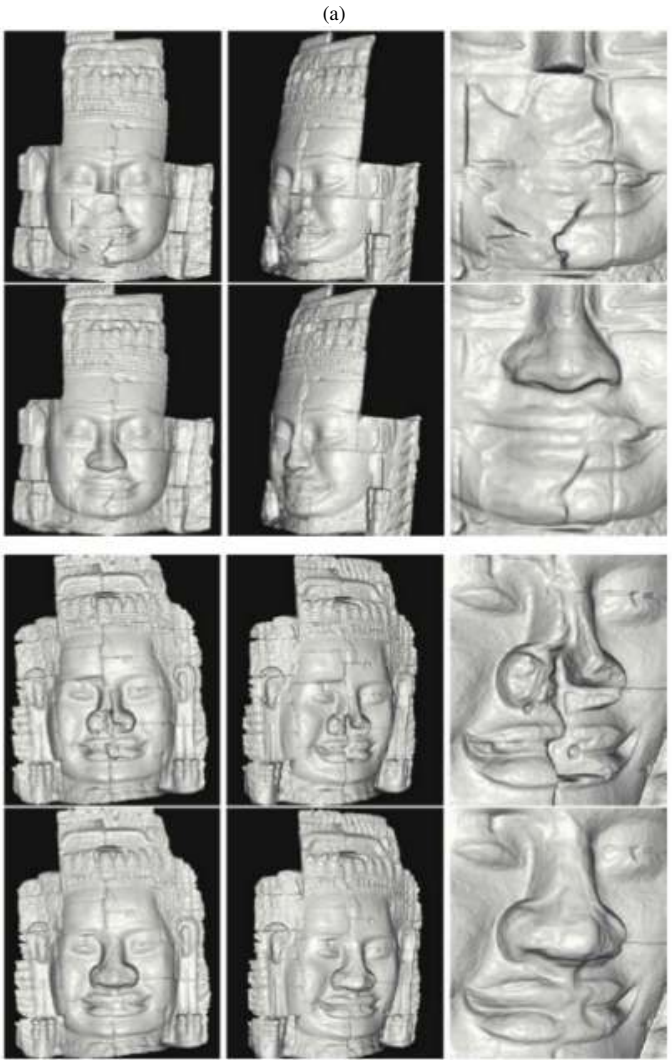

(b)

Figure 27: Restoration of statues from many-to-many analysis. (a) the basic idea behind restoration of collection of object. After aligning of all $3 D$ models, dense correspondences are found. A vector representation of each input shape is computed from the correspondence sets, and matrix recovery is applied to restore missing parts; $(b)$ examples of restored results (upper model is the measured shape, while the bottom one is the restored output). Courtesy of $\mathrm{Lu}$ et al. [LZT*11].

tus with 3D scanned data. It proposes a framework of automatic object categorization, where distinguishing regions are simultaneously detected. In particular, they employ a fully data-driven categorization method to extract distinguishing regions with the aim of recognizing the style of forehead hair. In a similar way, Bevan et al. [BLMT*14] perform a many-to-many morphometric analysis and comparative taxonomy of the ear shapes in a large set of China's terracotta warriors. They acquire the statue by using Structure-frommotion technique and produce a detailed 3D model of each warrior's head. Then, they standardised the geometry of each ear in order to make it suitable for comparative purposes, by defining a canonical size, orientation and point density. They employ an ICP-based framwork to register all ear items, in order to compute a dense distance matrix between each pair of ears. Dissimilarity study applied to this database lead to some conclusions about workshop microstyle, e.g., distinct workshop locales or worker origins.

Hinton et al. [HHML11] use geometric comparison to infer the temporal sequence of the production of a series of busts of Benjamin Franklin. They considered 3D scans of marble, terra cotta and plaster models. By comparing cross sections, and taking into account the nature of the types of production, they justify a particular temporal sequence for when the previously undated busts were produced.

An on going project [YY14] acquired a collection of several busts sculpted by Louis-François Roubiliac portraying Alexander Pope; a triangulation laser scanner has been used to generate the high resolution $3 \mathrm{D}$ digital models. The purpose of the project is a many-to-many combinatorial comparison of the busts' shapes by using geometrical analysis tools, in order to provide scholars with a more quantitative information about the creative process of 18 th century sculptors. In this case, collection analysis performed on this dataset will overlap with the $\mathrm{CH}$ application of interpretation. In other words, collection analysis will help to shed light on hypotheses about the order in which those busts were created, and about the "relative roles the busts have played in Roubiliac's praxis" [YY14].

\subsection{Discussion}

In Fig. 28 we present the distribution of the paper in the \# Objects and $\mathbf{C H}$ Application axes. We can see how all $\mathrm{CH}$ applications benefit from an analysis at a macro level. Unlike micro- or meso- approaches, since global shape, preservation condition and nature of ancient artifacts have a high variability at macro scale, even in the same category, most projects deal just with a single object. Moreover, sometimes they present analyses tailored to a particular $\mathrm{CH}$ test case. The studied items range from small coins to buildings, and various types of 3D acquisitions are employed, including close-range laser scanning, photogrammetry, time-of-flight scanning, and computational tomography.

Few works present results where many 3D models are compared to one another. In the restoration pipeline geometrical information of a set of object is used to digitally restore damaged or missing parts. Through time, the diver- 

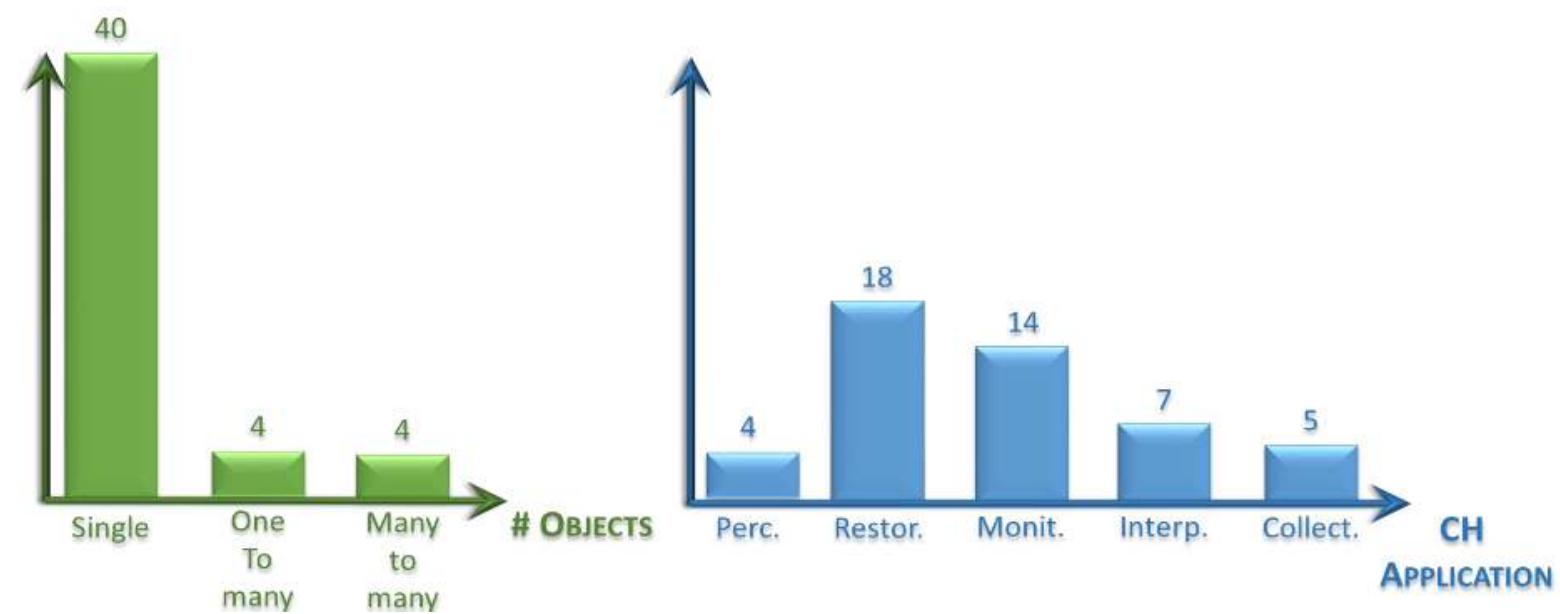

Figure 28: Macro-scale distribution. All the techniques are spread over the CH Application axis. However, due to the scale of the analysis, so far they focus mostly on the study of a single type of object or even a particular isolated test case (see \# Objects distribution).

sity of data acquired (images, 3D, etc) are used in a one-tomany framework for monitoring purposes. As expected, data collection benefits from the acquisition, characterization and retrieval algorithms applied to sets of $\mathrm{CH}$ items, such as statues organized in big databases.

\section{Multi-scale techniques}

In order to classify each paper, our taxonomy takes into consideration the predominant geometric scale at which the study is performed. However, many works are a weighted combination of two or more levels of analysis. In this section we provide the reader with few but most relevant examples of these techniques, coming from all micro-, meso, and macro-scale categories. They show how $\mathrm{CH}$ applications highly benefit from the design of hybrid algorithms.

In general, restoration by re-assembling fragments is based on the computation of micro-scale features, or on meso-scale assumptions about the local geometrical behaviour. Nevertheless, since the ultimate goal is to reconstruct the final, entire work of art, they need to drive the matching of the parts from a global point of view as well (e.g., $\left[\mathrm{HFG}^{*} 06\right]$ in section 4 ). At the end of the pipeline, each single match should be globally consistent with all the others. This aspect is mostly related to the idea of the object as a whole, which is the main characteristic of macro-scale methods. Another representative multi-scale method comes from the meso-scale category, restoration class [ $\left.\mathrm{PMW}^{*} 09\right]$. In that work, authors repair damaged, old photos by employing analysis at all geometric levels. First, they extract a geometry based signal of the photo surface by computing pseudo-normals (i.e., normal along one axis only) from an adapted Photometric Stereo technique. While the pseudo- normals are a micro-scale primitive attributes (here the primitives are image pixels), they are used for the meso-scale analysis task of extracting damaged large sub-regions. Finally, in order to repair those regions, they employ a statistical image infilling, which considers the global behaviour of color signal in the image, i.e., a macro-scale analysis. While the main contribution of the paper is the damaged pixel retrieval (meso), the final result wouldn't be possible without a processing at micro and macro level. The last example is about collection analysis performed at macro-scale. Lu et al. [LZZ ${ }^{*}$ 13] built a framework for the characterization and the comparison of entire models of portrait sculptures. Although their distance tensor is a macro-scale representation of the relative distances between each portrait with all the others, they use non-rigid alignment pipeline and distance metrics based on correspondences at micro level. Further, the final classification result has been undertaken by focussing only to one sub-region (meso-scale) of the portraits, i.e., their forehead hair.

Athough it is useful to organize the literature by defining clear taxonomy axes and categories, very few papers are focused only on a specific geometric scale; CG researchers should rely on this factor when designing new algorithms, frameworks or process pipelines, in order to properly balance the amount of different levels of analysis.

\section{Conclusions and Future work}

For each individual class micro-, meso-, or macro-scale, we have shown the distribution of the papers along the \# Objects and $\mathbf{C H}$ Application axes. Here we analyze how those distributions vary when we move along the Geometric Scale direction (Fig. 29). In Fig. 29(a) we depict how the distribu- 


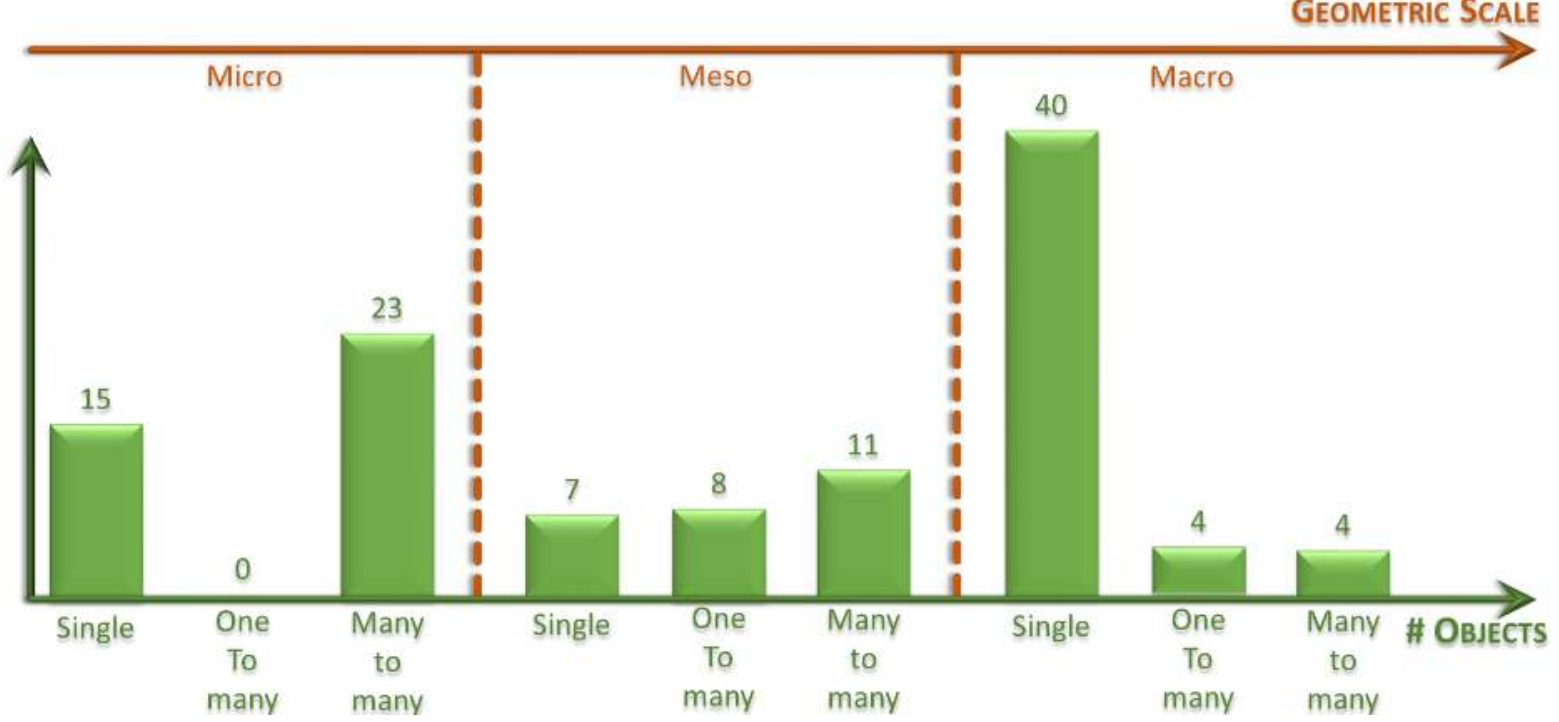

(a)

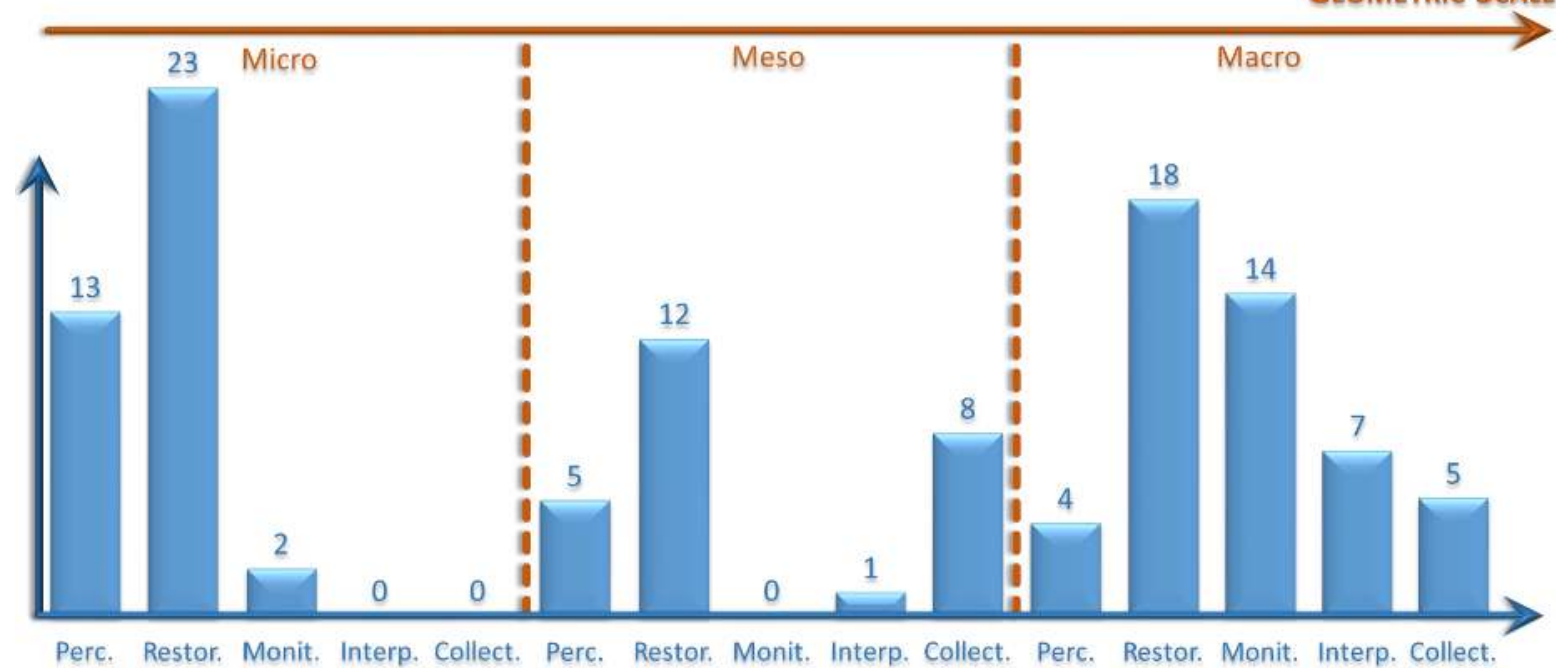

Perc. Restor. Monit. Interp. Collect. Perc. Restor. Monit. Interp. Collect. Perc. Restor. Monit. Interp. Collect.
CH

Application

(b)

Figure 29: Geometric Scale vs \# Objects and CH Application. We show the distribution of works in the \# Objects and $\mathbf{C H}$ Application axes when moving from micro- to macro-scale classes.

tion of papers in the \# Objects axis varies as we move from micro- to macro-scale in the Geometric Scale taxonomy. Since at small scales geometrical features are "more similar", it is possible to perform comparisons between a large number of different objects, such as "very-variable-shape" fragments of the same broken work of art; thus, a lot of contributions belong to the many-to-many class in the microscale analysis. On the other hand, the bigger the size of the studied area, the more peculiar and unique is its geometry, and the majority of methods dealing with the overall shape macro-scale tend to focus on the analysis of a single artifact. As a result, the meso-scale techniques equally cover all the groups, while, as we move from micro to macro, the density of the papers goes from many-to-many to single. A similar correlation is visible between Geometric Scale and CH Application as well (Fig. 29(b)). While macro-scale covers all kind of $\mathrm{CH}$ applications due to the high diversity of objects, micro- and meso- approaches study objects at a scale where 


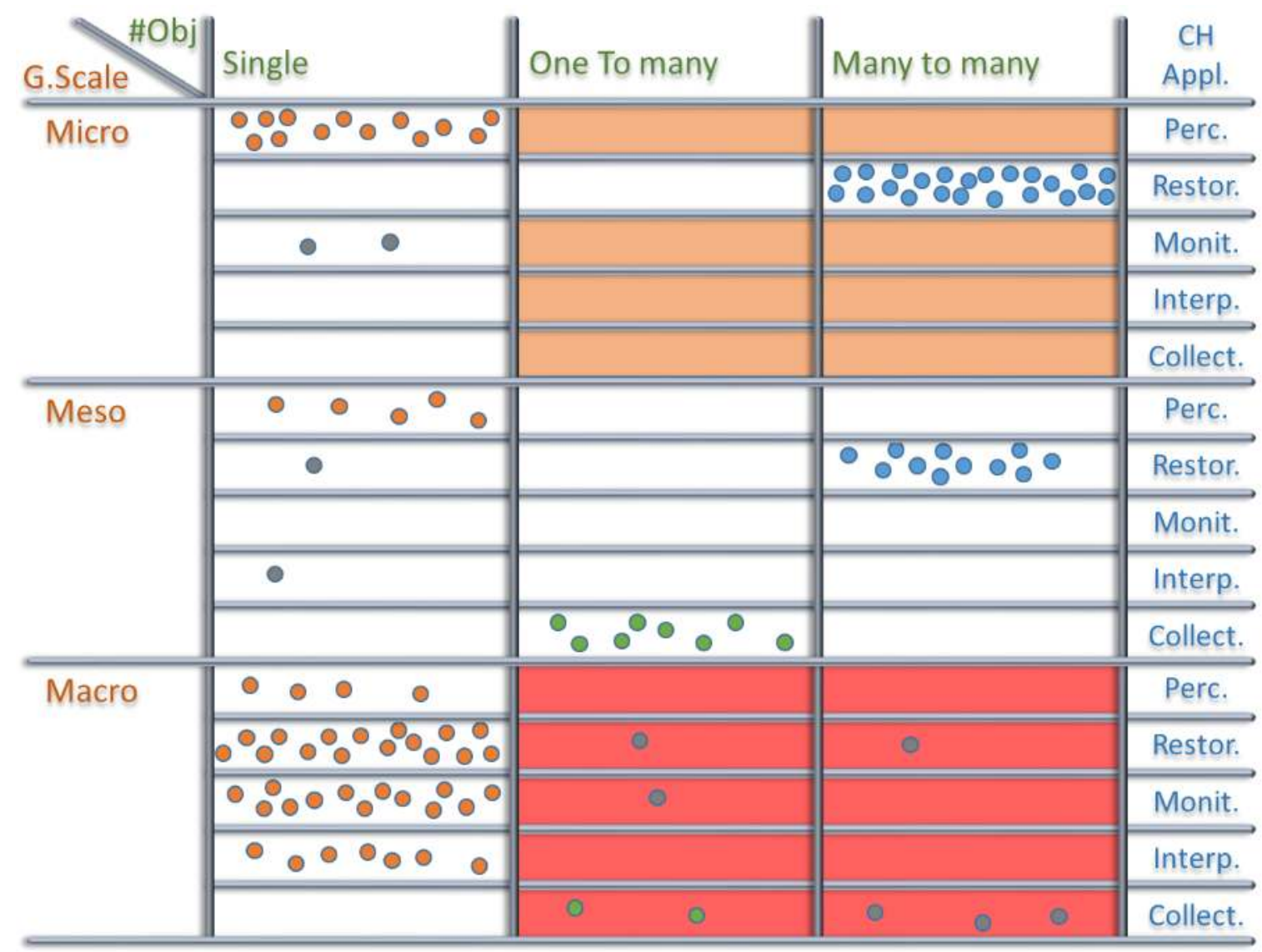

Figure 30: Clusters of papers and lacks in the literature. We identify three main clusters in the available literature: (a) Cluster $\# 1$ is related to restoration (blue dots) and Cluster \#2 is the set of all CH applications focused on single object analysis (orange dots); (b) A smaller cluster (Cluster \#3) is the group of works related to databases and collection analysis (green dots). The other un-clustered papers are marked as gray dots. From this table, we can see some gaps in the literature: some empty regions in the taxonomy are possibly related to ill-posed problems, e.g., orange empty cells. Conversely, although a lot of large sets of $3 D$ models exist, a small number of contributions deal with their analysis and study as a collection of similar artworks (red background cells).

they tend to look similar, so only few particular applications benefit from this effect. The two big clusters of methods in micro-scale analysis are restoration and perception. The former is mostly related to the task of re-assembling fragmented objects, while the latter deals with the computation of microscale signal to improve detail visibility. Apart from one single method in the monitoring, in general, no other $\mathrm{CH}$ applications are covered. The majority of the papers in the mesoscale are adaptations of micro-scale approaches in the case of an a-priori knowledge of the object type. Those are in the restoration and perception classes. The analysis at a bigger scale than micro, which takes into account sub-regions of an artwork, provides a tool to compare similar objects with one another. For this reason, the meso-scale techniques well cover the collection analysis class; all these papers are re- lated to the one-to-many category in the \# Objects axis. Another way to depict the distribution of the papers w.r.t. all the three axes is used in table 1, which highlights and improves the understanding on how strong is the correlation in the literature between the scale of the analysis and the number of samples involved. We re-organized this table in the Fig. 30, in order to study a possible cross-grouping of those papers from different categories. We can immediately identify two big clusters. The Cluster \#1 is related in general to restoration. The core of this cluster (blue dots) consists in all the micro and many-to-many techniques; we can extend this set with all the meso and many-to-many methods, which have the same theoretical background, but use more information from the object type. Conversely, the second cluster (Cluster \#2) is the wide spectrum of $\mathrm{CH}$ applications fo- 
cused on the single object analysis (orange dots). At macroscale level we can recognize the core of this set, but we can include the approaches in the micro-scale and perception categories; those increase the micro detail readability, but the ultimate goal is to allow scholars to read and better perceive the entire object as a whole. We identify another small cluster (Cluster \#3) as the group of works related to databases, i.e., classification, indexing and query $\mathrm{CH}$ items by geometric analysis and processing (green dots). Of course they rely on object types, so we found no paper in the microscale category. They are related to meso- or macro-scale, and to collection analysis. The un-clustered contributions are marked as gray dots. From another point of view, these three groups help us to identify empty regions in the taxonomy, regions where the available literature lacks. Some of those regions are related to maybe ill-posed problems (e.g., orange empty cells). However, although in recent years a lot of effort has been undertaken to acquire large collections of objects [BGMP13, BJM*14, YY14], we can say that only a very small number of works deal with the analysis of set of artworks (red background cells). This lack of comparative studies is the most important element that arises from our survey to give a direction for future CG analyses applied to $\mathrm{CH}$.

Another important element in the presented literature is the distribution of works over the time. Most papers that perform geometrical analysis were published in the last five years. This is due to the fact that $3 \mathrm{D}$ acquisition procedures and instruments are very mature now, and they are available at relatively low cost compared with a decade ago. These capture pipelines are faster and easier to operate, and the size, accuracy and resolution of digital models are increasing day by day. Micro and meso analyses benefit from the high accuracy and resolution of 3D models, which are acquired with a sub-millimeter precision, while macroscale techniques exploit the capabilities of fast laser scanner and photogrammetry to easily acquire objects ranging from small items to huge archaeological sites or buildings. Moreover, computational time has been greatly reduced in the last decade due to a great improvement of the available low cost hardware and the development of efficient parallel algorithms.

Future work will focus on two branches of the presented literature, i.e., the algorithmic and computational improvement of the analysis techniques, and the design of tools helping scholars to take the highest advantages from those approaches for their own research. Perception enhancement methods will study a compressed representation of extracted surface details, in order to allow fast access; for instance, combining stylistic analysis and sketch-based outputs will allow scholars to avoid manipulating high resolution 3D models and will support fast interpretation tasks. Although geometry reassembling for restoration was studied in many different practical test cases, it remains a challenging topic with open problems. For example, it would be interesting to study different materials and causes of failure, which produce different classes of fragments in terms of the corresponding fracture pattern. Another interesting topic would be to incorporate observed statistics of fractures and matches in a machine learning framework; classifiers could learn from previously assembled pieces or objects, and they could be applied to new artifacts. The quality of 3D models for monitoring is another issue when the acquisition time is a critical element, such as for archaeological sites during excavation activities. Possible future improvements in this field are related to the output data from Structure from Motion (SfM) algorithm in terms of geometry and color. Nowadays SfM pipelines and photo blending techniques are evolving fast to allow high resolution results comparable to models from 3D laser scanning, and it is important to investigate which of them are more suitable for $\mathrm{CH}$ purposes. The use of these procedures within an $\mathrm{CH}$ scenario poses also the problem of defining a formal guideline for archeologists; from their testing and feedback, some relevant issues must be adapted in the acquisition pipelines and in the visualization tools, such as the placement of markers, photographic campaign strategies, the processing and the integration of the data, and annotation capabilities. A similar challenge is the design of a tool to assist researchers during the interpretation task; apart from specific studies, a generally defined framework is required to validate hypotheses or to solve debates by using objective analyses based on geometric measurements. All of these are open problems that require a lot of interaction between scholars in $\mathrm{CH}$ and computer scientists. As the number of 3D models hosted in digital libraries is increasing, there are increasing research opportunities to test and improve classification and browsing techniques for collection analysis. New databases and new mathematical representations will advance the state-of-the-art of this field, by allowing scholars to remotely perform more efficient and fast search. Finally, future work may populate some of the areas of the taxonomy we have presented that are currently empty. In addition, if we acquire the geometry of a model at very small absolute scale, the behaviour of the fine scale shape will affect the appearance of the object itself. For this reason, some approaches that analyze the material appearance might be strongly related to the geometrical signal, and they might be included in the taxonomy whitin the microscale analysis class or in a new separate category.

\footnotetext{
Acknowledgments. This work was partially supported by the Digitally Enabled Scholarship with Medieval Manuscripts (DESMM) project funded by the Mellon Foundation (ydc2.yale.edu/). This work was also supported by the UCL EngD VEIV Centre for Doctoral Training and by London Metropolitan Archives. We also acknowledge the contribution of Sardinian Regional authorities and the support for H. Rushmeier from US National Science Foundation grant IIS-1302267.
} 


\section{References}

[AADC*07] ARIAS P., ARMESTO J., Di-CAPUA D., GonzÁlez-Drigo R., LOREnZO H., PÉREZ-GRACIA V.: Digital photogrammetry, GPR and computational analysis of structural damages in a mediaeval bridge. Engineering Failure Analysis 14, 8 (2007), 1444-1457. 4, 16

[ACCLA07] ARIAS P., Carlos CaAmaño J., Lorenzo H., ARMESTO J.: 3D modeling and section properties of ancient irregular timber structures by means of digital photogrammetry. Computer-Aided Civil and Infrastructure Engineering 22, 8 (2007), 597-611. 4, 16

[Add00] AdDISON A.: Emerging trends in virtual heritage. MultiMedia, IEEE 7, 2 (2000), 22-25. 1

[AHLO05] Arias P., Herráez J., Lorenzo H., OrdóÑez C.: Control of structural problems in cultural heritage monuments using close-range photogrammetry and computer methods. Computers \& Structures 83, 21-22 (2005), 1754-1766. 4, 16

[AIK13] ARTAL-ISBRAND P., KLAUSMEYER P.: Evaluation of the relief line and the contour line on Greek red-figure vases using reflectance transformation imaging and three-dimensional laser scanning confocal microscopy. Studies in Conservation 58, 4 (2013), 338-359. 4, 6

[Aim03] AimAtShape: Digital shape repository. More info on: http://aimatshape.net, 2003. 5

[AMK14] Altantsetseg E., Matsuyama K., Konno K.: Pairwise matching of 3D fragments using fast Fourier transform. The Visual Computer 30, 6-8 (2014), 929-938. 4, 7

[ARPLA10] ARMesto J., RocA-PARdiñas J., LORENZO H., ARIAS P.: Modelling masonry arches shape using terrestrial laser scanning data and nonparametric methods. Engineering Structures 32, 2 (2010), 607-615. 4, 16

[ASC*13] Arbace L., Sonnino E., Callieri M., DellepiANE M., FABbri M., Idelson A. I., SCOPIGNo R.: Innovative uses of $3 \mathrm{D}$ digital technologies to assist the restoration of a fragmented terracotta statue. Journal of Cultural Heritage 14, 4 (2013), $332-345.4,8$

[Ask12] Ask C.: Interpreting in 3D. Master's thesis, Lund University, Sweden, 2012. 2

[BCDA*05] BARnetT T., Chalmers A., DíAZ-Andreu M., Ellis G., LOnghurst P., Sharpe K., Trinks I.: 3D laser scanning for recording and monitoring rock art erosion. International newsletter on rock art. 41 (2005), 25-29. 4, 16

[BCP*07] Brunetti M., Callieri M., Pizzo B., Montani C., Pingi P., Scopigno R.: Using 3D scanning to monitor wood deformations and to evaluate preservation strategies, 2007. 4,6

[BGMP13] Bettio F., Gobbetti E., Merella E., Pintus $\mathrm{R}$.: Improving the digitization of shape and color of 3D artworks in a cluttered environment. In Proc. Digital Heritage (October 2013), pp. 23-30. Best Paper Award. 22

[BJM*14] Bettio F., Jaspe Villanueva A., Merella E., Marton F., Gobbetti E., Pintus R.: Mont'e Scan: Effective shape and color digitization of cluttered 3D artworks. ACM Journal on Computing and Cultural Heritage 8, 1 (2014), Article 4. 22

[BKMK10] Bechtold S., KRÖMKeR S., MARA H., KRATZMÜLlER B.: Rollouts of fine ware pottery using high resolution 3D meshes. In Proceedings of the 11th International conference on Virtual Reality, Archaeology and Cultural Heritage (2010), Eurographics Association, pp. 79-86. 4, 13
[BLD*12] BRown B., LAKen L., Dutré P., VAn Gool L., RUSINKIEWICZ S., WEYRICH T.: Tools for virtual reassembly of fresco fragments. International Journal of Heritage in the Digital Era 1, 2 (2012), 313-330. 8

[BLMT*14] BEVAN A., Li X., MARTINÓN-Torres M. Green S., Xia Y., Zhao K., Zhao Z., Ma S., CaO W., REHREN T.: Computer vision, archaeological classification and china's terracotta warriors. Journal of Archaeological Science (2014). 4, 18

[BP10] Bourdeu A., PizTalis D.: Geometric morphometrics for provenance determination of gallo-roman white clay figurines. In Proceedings of the 11th International conference on Virtual Reality, Archaeology and Cultural Heritage (2010), Eurographics Association, pp. 25-31. 4, 17

[BRM*02] Bernardini F., Rushmeier H., Martin I. M., Mittleman J., TAubin G.: Building a digital model of Michelangelo's Florentine Pietà. Computer Graphics and Applications, IEEE 22, 1 (2002), 59-67. 15, 17

[BRTL88] BOUlanger P., RiouX M., TAYlor J., LivingSTONE F.: Automatic replication and recording of museum artifacts. In Analysis and examination of an art object by imaging technique. Tokyo National Research Institute of Cultural Properties, 1988, pp. 131-147. 1

[BS04] BROWN M. S., SEALES W. B.: Image restoration of arbitrarily warped documents. Pattern Analysis and Machine Intelligence, IEEE Transactions on 26, 10 (2004), 1295-1306. 4, 14

[BSY*07] BRown M. S., Sun M., YAnG R., Yun L., Seales W. B.: Restoring 2D content from distorted documents. Pattern Analysis and Machine Intelligence, IEEE Transactions on 29, 11 (2007), 1904-1916. 4, 14

[BTFN*08] Brown B., Toler-Franklin C., Nehab D., Burns M., Vlachopoulos A., Doumas C., Dobin D., RUSINKIEWICZ S., WEYRICH T.: A system for high-volume acquisition and matching of fresco fragments: Reassembling Theran wall paintings. ACM Trans. on Graphics (Proc. SIGGRAPH 2008) 27, 3 (2008), 84:1-84:9. 4, 7, 9

[BV12] Belenguer C. S., Vidal E. V.: Archaeological fragment characterization and 3D reconstruction based on projective gpu depth maps. In Virtual Systems and Multimedia (VSMM), 2012 18th International Conference on (2012), IEEE, pp. 275282. 4,8

[CCG*04] Callieri M., Cignoni P., Ganovelli F., ImPoCo G., Montani C., Pingi P., Ponchio F., Scopigno R.: Visualization and $3 \mathrm{D}$ data processing in the david restoration. Computer Graphics and Applications, IEEE 24, 2 (2004), 16-21. 4, 15

[CDD*11] Callieri M., Dell'Unto N., Dellepiane M., Scopigno R., Soderberg B., LARSSON L.: Documentation and interpretation of an archeological excavation: an experience with dense stereo reconstruction tools. In Proceedings of the 12th International conference on Virtual Reality, Archaeology and Cultural Heritage (2011), Eurographics Association, pp. 3340. 4,16

[CFB*13] Canciani M., Falcolini C., Buonfiglio M., Pergola S., Saccone M., Mammì B., Romito G.: A method for virtual anastylosis: The case of the Arch of Titus at the Circus Maximus in Rome. ISPRS Annals of Photogrammetry, Remote Sensing and Spatial Information Sciences II-5/WI (2013), 61-66. 4, 12

[CGPS08] Cignoni P., Gobbetti E., Pintus R., Scopigno R.: Color enhancement for rapid prototyping. In Proceedings of the 9th International conference on Virtual Reality, Archaeology 
and Cultural Heritage (2008), Eurographics Association, pp. 916. $4,5,6,13$

[CLE12] COHEN F., LiU Z., EzGi T.: Virtual reconstruction of archaeological vessels using expert priors \& intrinsic differential geometry information. Computers \& Graphics (2012). 4, 12, 13

[DACC05] Devereux B., Amable G., Crow P., Cliff A.: The potential of airborne lidar for detection of archaeological features under woodland canopies. Antiquity 79, 305 (2005), 648660. 4,16

[DB06] Doneus M., BRIESE C.: Digital terrain modelling for archaeological interpretation within forested areas using fullwaveform laserscanning. In Proceedings of the 7th International conference on Virtual Reality, Archaeology and Intelligent Cultural Heritage (2006), Eurographics Association, pp. 155-162. 4,16

[DCF*07] Dellepiane M., CAllieri M., Fondersmith M., Cignoni P., SCOPIGNo R.: Using 3D scanning to analyze a proposal for the attribution of a bronze horse to Leonardo da Vinci. In Proceedings of the 8th International conference on Virtual Reality, Archaeology and Intelligent Cultural Heritage (2007), Eurographics Association, pp. 117-124. 4, 17

[DDUC*13] Dellepiane M., Dell Unto N., Callieri M., LINDGREN S., SCOPIGNo R.: Archeological excavation monitoring using dense stereo matching techniques, May-June 2013. 4,16

[DRDSH*14] De Reu J., De Smedt P., Herremans D., VAn Meirvenne M., LALOo P., De ClercQ W.: On introducing an image-based 3D reconstruction method in archaeological excavation practice. Journal of Archaeological Science 41 (2014), 251-262. 4, 16

[DST05] Docchio F., SAnsoni G., Trebeschi M.: Inspection, 3D modelling, and rapid prototyping of cultural heritage by means of a 3D optical digitiser. In Optical Metrology (2005), International Society for Optics and Photonics, pp. 58570D58570D. 4, 17

[FdBBB*13] Fratus de Balestrini E., Ballarin M., BALLetti C., Buttolo V., Gottardi C., Guerra F., Mander S., PILOT L., VERnier P.: Survey methods for earthquake damages in the "camera degli sposi" of mantegna (mantova). ISPRS - International Archives of the Photogrammetry, Remote Sensing and Spatial Information Sciences XL-5/W2 (2013), 265-270. 4, 16

[FdCP*08] Fantini M., De Crescenzio F., Persiani F., BeNAZZI S., GRUPPIONI G.: 3D restitution, restoration and prototyping of a medieval damaged skull. Rapid Prototyping Journal 14, 5 (2008), 318-324. 4, 16

[FMOS11] Fatuzzo G., Mussumeci G., Oliveri S., SeQUENZIA G.: The "guerriero di castiglione": reconstructing missing elements with integrated non-destructive 3D modelling techniques. Journal of Archaeological Science 38, 12 (2011), 3533-3540. 4, 15

[FRB*13] Fiorillo F., REMONdino F., BARBa S., SANTORiello A., De Vita C. B., Casellato A.: 3D digitization and mapping of heritage monuments and comparison with historical drawings. ISPRS Annals of Photogrammetry, Remote Sensing and Spatial Information Sciences II-5/W1 (2013), 133-138. 4,17

[FSTF*11] Funkhouser T., Shin H., TOler-Franklin C., Castañeda A. G., Brown B., Dobkin D., Rusinkiewicz S., WEYRICH T.: Learning how to match fresco fragments. $J$. Comput. Cult. Herit. 4, 2 (Nov. 2011), 7:1-7:13. 4, 7, 8

[GCBR*11] GARcía CASTAÑEdA A., Brown B. J.,
Rusinkiewicz S., Funkhouser T. A., Weyrich T. Global consistency in the automatic assembly of fragmented artefacts. In VAST (2011), pp. 73-80. 4, 8

[GCJ*07] Gorisse D., CORD M., JORdAN M., PhILIPPFoliguet S., PREcioso F.: 3d content-based retrieval in artwork databases. In 3DTV Conference, 2007 (2007), IEEE, pp. 14. 4,17

[GTSK13] Gilboa A., TAl A., Shimshoni I., Kolomenkin M.: Computer-based, automatic recording and illustration of complex archaeological artifacts. Journal of Archaeological Science 40, 2 (2013), 1329-1339. 4

[GWS*09] GUNAWARdANE P., WANG O., Scher S., Rickards I., DAVIS J., Malzbender T.: Optimized image sampling for view and light interpolation. In Proceedings of the 10th International conference on Virtual Reality, Archaeology and Cultural Heritage (2009), Eurographics Association, pp. $93-100.4,6$

[HFG*06] HuAng Q.-X., FlÖry S., GElFAND N., HofEr M., PotTMAnN H.: Reassembling fractured objects by geometric matching. In ACM Transactions on Graphics (TOG) (2006), vol. 25, ACM, pp. 569-578. 4, 6, 7, 19

[HHMl11] Hinton J., Houdon J. A., Meighan M., Lins P. A.: Encountering Genius: Houdon's Portraits of Benjamin Franklin. Philadelphia Museum of Art, 2011. 18

[HMD*10] Happa J., Mudge M., Debattista K., Artusi A., GonçAlves A., CHAlmers A.: Illuminating the past: state of the art. Virtual reality 14, 3 (2010), 155-182. 2

[HRM*09] Hess M., Robson S., Millar F. S., Were G., HVIDING E., BERG A. C.: Niabara-the western solomon islands war canoe at the british museum-3D documentation, virtual reconstruction and digital repatriation. In 15th International Conference on Virtual Systems and Multimedia (VSMM), 2009 (2009), IEEE, pp. 41-46. 4, 15

[HW11] Hameeuw H., Willems G.: New visualization techniques for cuneiform texts and sealings. Akkadica 132 (2011), 163-178. 4, 5

[IT11] Itskovich A., TAL A.: Surface partial matching and application to archaeology. Computers \& Graphics 35, 2 (2011), 334-341. 4, 11, 12

[KDSK10] Kleber F., Diem M., SAblatnig R., KAMPel M.: Proposing features for the reconstruction of marble plates of ephesos. In Virtual Systems and Multimedia (VSMM), 2010 16th International Conference on (2010), IEEE, pp. 328-331. 4, 8

[KM03] KAMPEL M., Melero F. J.: Virtual vessel reconstruction from a fragment"s profile. In Proceedings of the 4th International conference on Virtual Reality, Archaeology and Intelligent Cultural Heritage (2003), Eurographics Association, pp. 79-88. 4,8

[KO13] KIKUCHI T., OGIHARA N.: Computerized assembly of neurocranial fragments based on surface extrapolation. Anthropological Science 121, 2 (2013), 115-122. 4, 12

[KOC*14] Knibbe J., O’Hara K. P., Chrysanthi A., MarShALl M. T., BENNETT P. D., EARL G., IZADI S., FRASER M.: Quick and dirty: streamlined 3D scanning in archaeology. In Proceedings of the 17th ACM conference on Computer supported cooperative work \& social computing (2014), ACM, pp. 13661376. 4, 16

[KPC10] Koutsoudis A., Pavlidis G., Chamzas C.: Detecting shape similarities in 3D pottery repositories. In Semantic Computing (ICSC), 2010 IEEE Fourth International Conference on (2010), IEEE, pp. 548-552. 4, 11, 12 
[KRF*14] KIM M. H., RushmeIER H., FFrenCH J., PASSERI I., TIDMARSH D.: Hyper3D: 3D graphics software for examining cultural artifacts. J. Comput. Cult. Herit. 7, 3 (Feb. 2014), $14: 1-14: 19.16$

[KS04] KAMPEL M., SABLATNIG R.: 3D puzzling of archeological fragments. In Proceedings of 9th Computer Vision Winter Workshop (2004). 4, 12

[KS08] KARASIK A., SMILANSKY U.: 3D scanning technology as a standard archaeological tool for pottery analysis: practice and theory. Journal of Archaeological Science 35, 5 (2008), 1148-1168. 4, 11

[KS11] Karasik A., Smilansky U.: Computerized morphological classification of ceramics. Journal of Archaeological Science 38, 10 (2011), 2644-2657. 4, 11

[KST08] Kolomenkin M., Shimshoni I., TAl A.: Demarcating curves for shape illustration. In ACM Transactions on Graphics (TOG) (2008), vol. 27, ACM, p. 157. 4

[KST11] Kolomenkin M., Shimshoni I., Tal A.: Prominent field for shape processing and analysis of archaeological artifacts. International Journal of Computer Vision 94, 1 (2011), 89-100. 3,4

[Lan13] LANDON G. V.: Automatic photometric restoration of historical photographic negatives. In Proceedings of the 2 nd International Workshop on Historical Document Imaging and Processing (2013), ACM, pp. 1-7. 4, 14

[LBM*13] LAYCOCK S. D., Bell G. D., Mortimore D. B., Greco M. K., CorPs N., FInKLE I.: Combining X-ray microct technology and 3D printing for the digital preservation and study of a 19th century cantonese chess piece with intricate internal structure. J. Comput. Cult. Herit. 5, 4 (Jan. 2013), 13:1-13:7. 4,14

[LCM11] Lasaponara R., ColuzZI R., MAsini N.: Flights into the past: full-waveform airborne laser scanning data for archaeological investigation. Journal of Archaeological Science 38, 9 (2011), 2061-2070. 4, 16

[LD06] LEVY R., DAWsON P.: Reconstructing a thule whalebone house using 3D imaging. MultiMedia, IEEE 13, 2 (2006), 78-83. 4,15

[LlS06] Lin Y., LANDON G. V., Seales W. B.: Developing a framework for acquisition, preservation, and dissemination of 3D documents. In DEPARTMENT OF COMPUTER SCIENCE, UNIVERSITY OF KENTUCKY (2006), Citeseer. 4, 14

[LPC*00] LeVoy M., Pulli K., Curless B., RusinkiewiCZ S., Koller D., Pereira L., Ginzton M., Anderson S., Davis J., Ginsberg J., Shade J., FulK D.: The digital michelangelo project: 3D scanning of large statues. In Proc. SIGGRAPH (2000), pp. 131-144. 4, 5

[LS09] LANitis A., Stylianou G.: E-Restoration of faces appearing in cultural heritage artefacts. In 15th International Conference on Virtual Systems and Multimedia (VSMM), 2009 (2009), IEEE, pp. 15-20. 4, 17

[LSC08] Landon G. V., Seales W. B., Clarke D.: A new system to acquire and restore document shape and content. In Proceedings of the 5th ACM/IEEE International Workshop on Projector camera systems (2008), ACM, p. 12. 4, 14

[LW04a] LAUGERotTE C., WARZÉE N.: 3D orientation of archaeological fragments coming from a gothic spire. In Proceedings of the 5th International conference on Virtual Reality, Archaeology and Intelligent Cultural Heritage (2004), Eurographics Association, pp. 175-183. 4, 12

[LW04b] LaugerotTe C., WarzéE N.: An environment for the analysis and reconstruction of archaeological objects. In
Proceedings of the 5th International conference on Virtual Reality, Archaeology and Intelligent Cultural Heritage (2004), Eurographics Association, pp. 165-174. 8

[LYW*11] Li X., Yin Z., Wei L., WAN S., Yu W., Li M. Symmetry and template guided completion of damaged skulls. Computers \& Graphics 35, 4 (2011), 885-893. 4, 12

[LZT*11] Lu M., Zheng B., Takamatsu J., Nishino K., IKEUCHI K.: Preserving the Khmer smile: classifying and restoring the faces of bayon. In Proceedings of the 12th International conference on Virtual Reality, Archaeology and Cultural Heritage (2011), Eurographics Association, pp. 161-168. 4, 17, 18

[LZZ*13] LU M., ZHANG Y., ZHENG B., MASUdA T., ONO S., Oishi T., SENGOKU-HAga K., IKeUCHI K.: Portrait sculptures of Augustus: Categorization via local shape comparison. In 2013 International Congress on Digital Heritage, Marseille, France (2013). 4, 17, 19

[MBW*14] ManfRedi M., BeARMAN G., Williamson G., Kronkright D., Doehne E., JaCobs M., MARengo E.: A new quantitative method for the non-invasive documentation of morphological damage in paintings using rti surface normals. Sensors 14, 7 (2014), 12271-12284. 4, 6

[MGM*12] Muzzupappa M., Gallo A., Mattanò R., RugGIERO C., BRUNO F.: A complete morphological study of the right hand of bronzo" a" di riace. International Journal of Heritage in the Digital Era 1 (2012), 55-60. 4, 17

[MGW01] Malzbender T., Gelb D., Wolters H.: Polynomial texture maps. In Proceedings of the 28th annual conference on Computer graphics and interactive techniques (New York, NY, USA, 2001), SIGGRAPH '01, ACM, pp. 519-528. 4, 5,6

[Mil94] Miller G.: Efficient algorithms for local and global accessibility shading. In Proc. SIGGRAPH (New York, NY, USA, 1994), ACM, pp. 319-326. doi:10.1145/192161. 192244.5

[MK13] MARA H., KROMKER S.: Vectorization of 3Dcharacters by integral invariant filtering of high-resolution triangular meshes. In Document Analysis and Recognition (ICDAR), 2013 12th International Conference on (2013), IEEE, pp. 62-66. $3,4,5$

[MKJB10] MARA H., KRÖMKER S., JAKOB S., BREUCKMANN B.: Gigamesh and gilgamesh:-3D multiscale integral invariant cuneiform character extraction. In Proceedings of the 11th International conference on Virtual Reality, Archaeology and Cultural Heritage (2010), Eurographics Association, pp. 131-138. 3, 4

[MMSL06] Mudge M., Malzbender T., Schroer C., Lum M.: New reflection transformation imaging methods for rock art and multiple-viewpoint display. In Proceedings of the 7th International conference on Virtual Reality, Archaeology and Intelligent Cultural Heritage (2006), Eurographics Association, pp. 195-202. 4, 5, 6

[MPWC13] Mitra N. J., Pauly M., Wand M., Ceylan D.: Symmetry in 3D geometry: Extraction and applications. In Computer Graphics Forum (2013), vol. 32, Wiley Online Library, pp. 1-23. 2

[MRS10] Mellado N., Reuter P., Schlick C.: Semiautomatic geometry-driven reassembly of fractured archeological objects. In Proceedings of the 11th International conference on Virtual Reality, Archaeology and Cultural Heritage (2010), Eurographics Association, pp. 33-38. 4, 8

[MVSL05] Mudge M., Voutaz J.-P., Schroer C., Lum M.: Reflection transformation imaging and virtual representations of coins from the hospice of the grand St. Bernard. In Proceedings 
of the 6th International conference on Virtual Reality, Archaeology and Intelligent Cultural Heritage (2005), Eurographics Association, pp. 29-39. 4, 6

[MWZ*14] Mitra N., Wand M., Zhang H. R., Cohen-OR D., KIM V., HuANG Q.-X.: Structure-aware shape processing. Siggraph Course (2014). 2

[NFRM15] Nadel D., Filin S., Rosenberg D., Miller V.: Prehistoric bedrock features: recent advances in 3D characterization and geometrical analyses. Journal of Archaeological Science 53 (2015), 331-344. 4, 17

[OBP04] Owen R., Buhalis D., Pletinckx D.: Identifying technologies used in cultural heritage. In Proceedings of the 5th International conference on Virtual Reality, Archaeology and Intelligent Cultural Heritage (2004), Eurographics Association, pp. $155-163.2$

[ON11] OxhOLM G., Nishino K.: Reassembling thin artifacts of unknown geometry. In Proceedings of the 12th International conference on Virtual Reality, Archaeology and Cultural Heritage (2011), Eurographics Association, pp. 49-56. 4, 8

[ONHC13] Olsson P., Nysjo F., HiRsch J.-M., CARlbom I.: Snap-to-fit, a haptic 6 DOF alignment tool for virtual assembly. In World Haptics Conference (WHC), 2013 (2013), IEEE, pp. 205-210. 8

[PAE*12] PAPAODYSSEUS C., ARABAdJis D., Exarhos M., Rousopoulos P., Zannos S., Panagopoulos M., PAPAZOGLOU-MANIOUdAKI L.: Efficient solution to the 3D problem of automatic wall paintings reassembly. Computers \& Mathematics with Applications (2012). 4, 8

[PCMA08] Pitzalis D., Cignoni P., Menu M., Aitken G.: 3D enhanced model from multiple data sources for the analysis of the cylinder seal of ibni-sharrum. In Proceedings of the 9th International conference on Virtual Reality, Archaeology and Cultural Heritage (2008), Eurographics Association, pp. 79-84. 4, 13, 14

[PGCS10] Pintus R., Gobbetti E., Cignoni P., Scopigno R.: Shape enhancement for rapid prototyping. The Visual Computer 26, 6-8 (2010), 831-840. 4, 5, 6

[PK03] Papaionnnou G., Karabassi E.-A.: On the automatic assemblage of arbitrary broken solid artefacts. Image and Vision Computing 21, 5 (2003), 401-412. 4, 8

[PKT01] Papaionnnou G., Karabassi E.-A., Theoharis T.: Virtual archaeologist: Assembling the past. Computer Graphics and Applications, IEEE 21, 2 (2001), 53-59. 4, 8

[PKT02] Papaionnnou G., Karabassi E.-A., Theoharis T.: . Pattern Analysis and Machine Intelligence, IEEE Transactions on 24, 1 (2002), 114-124. 4, 7

[PMCS11] Pietroni N., Massimiliano C., Cignoni P., SCOPIGNO R.: An interactive local flattening operator to support digital investigations on artwork surfaces. Visualization and Computer Graphics, IEEE Transactions on 17, 12 (2011), 19891996. 4, 10

[PMW*09] Pintus R., Malzbender T., WANG O., Bergman R., NACHLieli H., RUCKEnStein G.: Photo repair and 3D structure from flatbed scanners. In VISAPP International Conference on Computer Vision Theory and Applications (2009). 4, 10, 11, 17, 19

[PPCS12] Palmas G., Pietroni N., Cignoni P., Scopigno R.: A practical framework for assembling fragmented objects. paper submitted to IEEE Computer Graphics and Applications on August (2012). 8

[PPY* 14] PINTUS R., PAL K., YANG Y., WEYRICH T., GOBBETTI E., RUSHMEIER H.: Geometric Analysis in Cultural Heritage. In The 12th Eurographics Worhshop on Graphics and Cul- tural Heritage - STARS Proceedings (2014), Eurographics Association, pp. 117-133. 2

[PSP*14] Pal K., Schüller C., Panozzo D., SorkineHORNung O., WEYRICH T.: Content-aware surface parameterization for interactive restoration of historical documents. In Computer Graphics Forum (2014), vol. 33, Wiley Online Library, pp. 401-409. 4, 10, 14, 15

[PTW13a] PAL K., TERRAS M., WEYRICH T.: 3D reconstruction for damaged documents: imaging of the great parchment book. In Proc. 2nd International Workshop on Historical Document Imaging and Processing (2013), ACM, pp. 14-21. 4, 10

[PTW13b] PAL K., TERRAS M., WEYRICH T.: Interactive exploration and flattening of deformed historical documents. In Computer Graphics Forum (2013), vol. 32, Wiley Online Library, pp. 327-334. 4, 10

[RCAS11] Riveiro B., CAamaño J., Arias P., SAnz E.: Photogrammetric 3D modelling and mechanical analysis of masonry arches: An approach based on a discontinuous model of voussoirs. Automation in Construction 20, 4 (2011), 380-388. 4, 16

[RCD*12] Ranzuglia G., Callieri M., Dellepiane M., Cignoni P., Scopigno R.: Meshlab as a complete tool for the integration of photos and color with high resolution $3 \mathrm{D}$ geometry data, 2012. 16

[REH06] REMONDINO F., EL-HAKIM S.: Image-based 3D modelling: A review. The Photogrammetric Record 21, 115 (2006), 269-291. 1

[RMK13] RIECK B., MARA H., KRÖMKER S.: Unwrapping highly-detailed 3D meshes of rotationally symmetric man-made objects. 4,13

[RRC*10] Reuter P., Riviere G., Couture N., Mahut S., ESPINASSE L.: Archeotui: Driving virtual reassemblies with tangible 3D interaction. Journal on Computing and Cultural Heritage (JOCCH) 3, 2 (2010), 4. 8

[RSdA*13] Riveiro B., Solla M., De Arteaga I., Arias P., MORER P.: A novel approach to evaluate masonry arch stability on the basis of limit analysis theory and non-destructive geometric characterization. Automation in Construction 31 (2013), 140-148. 4, 16

[SBG11] Stanco F., Battiato S., Gallo G.: Digital Imaging for Cultural Heritage Preservation: Analysis, Restoration, and Reconstruction of Ancient Artworks, vol. 3. CRC Press, 2011. 2

[SCC*11] SCOPIGno R., CAllieri M., Cignoni P., Corsini M., Dellepiane M., Ponchio F., Ranzuglia G.: 3D models for cultural heritage: Beyond plain visualization. Computer 44, 7 (2011), 48-55. 2

[SDF*12] Shin H., Doumas C., Funkhouser T., RusinkiewiCZ S., Steiglitz K., Vlachopoulos A., WEYRICH T.: Analyzing and simulating fracture patterns of Theran wall paintings. J. Comput. Cult. Herit. 5, 3 (Oct. 2012), 10:1-10:14. 4, 8

[SEG* 12] SÁ A. M., EChaVARria K. R., GrifFin M., Covill D., KAMINSKi J., ARNOLD D.: Parametric 3D-fitted frames for packaging heritage artefacts. In VAST: International Symposium on Virtual Reality, Archaeology and Intelligent Cultural Heritage (2012), The Eurographics Association, pp. 105-112. 4, 15

[SKN*12] SMith N. G., KarasiK A., NARAYANAN T., OLSON E. S., SMilansky U., LEVy T. E.: The pottery informatics query database: A new method for mathematic and quantitative analyses of large regional ceramic datasets. Journal of Archaeological Method and Theory (2012), 1-39. 4, 12

[SL04] SEALES W. B., LIN Y.: Digital restoration using volumetric scanning. In Digital Libraries, 2004. Proceedings of the 2004 
Joint ACM/IEEE Conference on (2004), IEEE, pp. 117-124. 4, 14,15

[SLMR14] Samko O., Lai Y.-K., Marshall D., Rosin P. L.: Virtual unrolling and information recovery from scanned scrolled historical documents. Pattern Recognition 47, 1 (2014), 248-259. 4, 14, 15

[SPK*13] SFIKAS K., PRATIKakis I., Koutsoudis A., SaVelonas M., Theoharis T.: . In New Trends in Image Analysis and Processing-ICIAP 2013. Springer, 2013, pp. 169178. 4

[SPK* 14] SFIKAS K., PRATIKaKis I., Koutsoudis A., SAVElonas M., TheOHARIS T.: . Multimedia Tools and Applications (2014), 1-15. 4

[SPS14] SaVElonas M. A., PratikaKis I., SFIKAS K.: . Eurographics Workshop on 3D Object Retrieval (2014). 4

[SS01] SchrödER P., SWELdENS W.: Digital geometry processing. In Frontiers of Engineering:: Reports on Leading-Edge Engineering From the 2000 NAE Symposium on Frontiers in Engineering (2001), National Academies Press, p. 41. 2

[SSB11] SCHMIDT N., SCHÜTZE R., Boochs F.: 3D sutra: Processing of scanned sutra inscriptions in China for analysis, interpretation and visualization. Revive the Past (2011), 81. 4, 10

[Sto09] STORK D.: Computer vision and computer graphics analysis of paintings and drawings: An introduction to the literature. In Computer Analysis of Images and Patterns, Jiang X., Petkov N., (Eds.), vol. 5702 of Lecture Notes in Computer Science. Springer Berlin Heidelberg, 2009, pp. 9-24. 2

[SVG09] Schueremans L., VAN GEnechten B.: The use of 3D-laser scanning in assessing the safety of masonry vaults:a case study on the church of saint-jacobs. Optics and Lasers in Engineering 47, 3 (2009), 329-335. 4, 16

[SYY*05] Sun M., YANG R., Yun L., LANDON G., SEALES B., BROWN M. S.: Geometric and photometric restoration of distorted documents. In Computer Vision, 2005. ICCV 2005. Tenth IEEE International Conference on (2005), vol. 2, IEEE, pp. 1117-1123. 4, 14

[TFBW*10] TOLER-Franklin C., Brown B., Weyrich T., FUnKhouser T., RUSINKIEWICZ S.: Multi-feature matching of fresco fragments. In ACM Transactions on Graphics (TOG) (2010), vol. 29, ACM, p. 185. 4, 7

[TFK*09] Thuswaldner B., FlÖRY S., KAlASEK R., HoFER M., HUANG Q.-X., THÜR H.: Digital anastylosis of the octagon in ephesos. Citeseer, 2009. 4, 12

[VVSB12] Vendrell Vidal E., SANChez Belenguer C.: Automatic archaeological fragment reconstruction using hierarchical depth maps. In Informatica (CLEI), 2012 XXXVIII Conferencia Latinoamericana En (2012), IEEE, pp. 1-10. 4, 7

[VVSB14] VENDREll-VidAl E., SÁNCHEZ-BELENGUER C.: A discrete approach for pairwise matching of archaeological fragments. Journal on Computing and Cultural Heritage (JOCCH) 7, 3 (2014), 15. 4

[WC04a] Willis A., COOPER D. B.: Alignment of multiple non-overlapping axially symmetric $3 \mathrm{D}$ datasets. In Pattern Recognition, 2004. ICPR 2004. Proceedings of the 17th International Conference on (2004), vol. 4, IEEE, pp. 96-99. 4, 12

[WC04b] WILlis A. R., COOPER D. B.: Bayesian assembly of 3D axially symmetric shapes from fragments. In Proceedings of the IEEE Computer Society Conference on Computer Vision and Pattern Recognition (CVPR) (2004), pp. 82-89. 4, 12

[WC08] Willis A. R., CoOper D. B.: Computational reconstruction of ancient artifacts. Signal Processing Magazine, IEEE 25, 4 (2008), 65-83. 4, 8, 12, 13

(c) 2015 The Author(s)

Computer Graphics Forum (c) 2015 The Eurographics Association and John Wiley \& Sons Ltd.
[WCV*03] WASSERMAN J., CAMIZ F. T., Verdon T., ROCKWell P., Amendola A.: Michelangelo's Florence Pietà. Princeton University Press, 2003. 4, 15, 17

[Woo79] Woodham R. J.: Photometric stereo: A reflectance map technique for determining surface orientation from image intensity. In 22nd Annual Technical Symposium (1979), International Society for Optics and Photonics, pp. 136-143. 5, 6, 10, 11

[WYLL11] WeI L., YU W., LI M., LI X.: Skull assembly and completion using template-based surface matching. In $3 D$ Imaging, Modeling, Processing, Visualization and Transmission (3DIMPVT), 2011 International Conference on (2011), IEEE, pp. 413-420. 4, 12

[YLL12] YU W., LI M., LI X.: Fragmented skull modeling using heat kernels. Graphical Models 74, 4 (2012), 140-151. 4, 7

[YWML11] Yin Z., Wei L., Manhein M., Li X.: An automatic assembly and completion framework for fragmented skulls. In Computer Vision (ICCV), 2011 IEEE International Conference on (2011), IEEE, pp. 2532-2539. 4, 8

[YY14] YDC2, YCBA: YCBA Pope bust scanning project. More info on: http://ydc2.yale.edu/ycba-pope-bust-scanningproject, 2014. 18,22

[ZL14] ZhANG K., Li X.: . Graphical Models 76, 5 (2014), 484-495. 4, 8

[ZLZ*13] Zhang Y., LU M., Zheng B., MASUdA T., Ono S., Oishi T., Sengoku-Haga K., IKeuchi K.: Classical sculpture analysis via shape comparison. In Culture and Computing (Culture Computing), 2013 International Conference on (2013), IEEE, pp. 57-61. 4, 17 